\title{
Diversidad vegetacional de la Reserva Nacional Malalcahuello, IX Región de Chile
}

Vegetational diversity of the $M$ alalcahuello $N$ ational Reserve, I $X^{\text {th }}$ Region of Chile

\author{
PABLO BECERRA ${ }^{1}$, GUSTAVO CRUZ ${ }^{2}$ \\ ${ }^{1}$ Depto. Ciencias Ecológicas, Facultad de Ciencias, Universidad de Chile, Casilla 653, Santiago, Chile. \\ E-mail: pbecerra@icaro.dic.uchile.cl ${ }^{2}$ Depto. Silvicultura, Facultad de Ciencias Forestales, \\ Universidad de Chile, Santiago, Chile.
}

\begin{abstract}
SUMMARY
Knowledge of the diversity and characteristics of phytosociological vegetation units of transitional regions between Mediterranean and temperate zones in Chile, particularly in the protected wild areas, is scarce. In this paper, the phytosociological units of the National Reserve "Malalcahuello", IX ${ }^{\text {th }}$ Region of Chile, are defined. The relative importance of elevation, exposition, and type of substrate for its formation is examined, along with a characterization of species and life form structure. Thirteen units were identified, which were formed as a consequence of elevation and substrate variation. The species richness varied between $21(10 \%)$ and $58(27.5 \%)$. The species similarities between units varied between $0 \%$ and $50.9 \%$, and the life form diversity varied between units in terms of the number of species and coverage per life form. The variation of species and life form diversity between units suggests that the incorporation of this data in SNASPE (Sistema Nacional de A reas Silvestres Protegidas del Estado en Chile) will improve the knowledge of biodiversity of Chile.
\end{abstract}

Key words: vegetation, R.N. Malalcahuello, species diversity, life forms, phytosociology.

\section{RESUMEN}

El conocimiento de la diversidad y características de las unidades vegetacionales fitosociológicas de la zona transicional entre las regiones mediterránea y templada de Chile, especialmente de sus áreas silvestres protegidas, es aún escaso. En este trabajo se definen las unidades vegetacionales fitosociológicas de la Reserva Nacional Malalcahuello, IX Región, Chile. Se examinan la importancia de la altitud, exposición y tipo de sustrato en determinar su formación, y se caracterizan su estructura de especies y formas de vida. Se encontraron 13 unidades fitosociológicas, las cuales se forman principalmente como consecuencia de la variación altitudinal y de sustratos. La riqueza de especies varía entre $21(10 \%)$ y $58(27,5 \%)$. La similitud de especies entre unidades varió entre el $0 \%$ y $50,9 \%$. La diversidad de formas de vida varió entre las unidades tanto al considerar el número de especies como la cobertura de cada forma de vida. La variación de la estructura de especies como de formas de vida entre las unidades vegetacionales sugiere que la incorporación de un mayor número de éstas en el SNASPE (Sistema $\mathrm{N}$ acional de A reas Silvestres Protegidas del Estado en Chile) permitiría mejorar su representatividad de la biodiversidad de Chile.

Palabras claves: vegetación, R.N. Malalcahuello, diversidad de especies, formas de vida, fitosociología.

\section{INTRODUCCION}

Uno de los componentes más importantes del paisaje de una zona geográfica determinada es la diversidad de unidades vegetacionales que éste presenta (Forman 1995). Estas pueden ser diferenciadas tanto por características fisionómicas como por la composición y abundancia de especies vegetales (B raun-Blanquet 1950, Whittake, 1973, Muller-Dombois \& Ellenberg 1974). Las unidades diferenciadas en función de la estructura florística corresponden a unidades vegetacionales fitosociológicas. En este caso, sólo a partir de unas pocas especies que se presentan en aproximada- 
mente los mismos sitios y que se segregan espacialmente de otras, denominadas especies diferenciales, permiten definir distintas unidades de vegetación (Braun-Blanquet 1950, M uller-D ombois \& Ellenberg 1974).

La formación de unidades vegetacionales fitosociológicas está determinada, en definitiva, por los procesos que establecen la existencia de especies diferenciales. Estos son la dispersión y sobrevivencia diferencial entre los distintos sitios de una zona. En el primer caso, algunas especies podrían tener una mayor probabilidad de dispersarse hacia determinados sitios y otras especies a otros (Howe \& Smallwood 1982). En el segundo caso, ciertas especies tendrían una mayor capacidad de sobrevivir y reproducirse en determinados sitios y otras especies en otros. Esto último podría deberse a diferentes tolerancias ecofisiológicas entre las especies a las condiciones físicas del ambiente (B raun-Blanquet 1950, W hittaker 1973, Stephenson 1990), o a interacciones bióticas, especialmente de competencia por recursos (Tilman 1988). La acción de estos mecanismos puede estar relacionada con factores indirectos del ambiente, entre los cuales han sido considerados como más importantes la posición topográfica y el tipo de sustrato. La variación topográfica, ya sea altitudinal o de exposición, puede modificar significativamente las condiciones de temperatura, humedad, viento, agentes bióticos de dispersión, etc. Por su parte, la variación de los tipos de sustratos puede estar asociada a cambios en la disponibilidad de nutrientes y niveles de drenaje (Braun-Blanquet 1950, W hittaker 1973).

L as unidades vegetacionales fitosociológicas de una zona deberían diferir en mayor o menor grado en su estructura de especies y formas de vida (B raun-B lanquet 1950, Whittaker 1972, 1973), conformando la diversidad del paisaje vegetacional del área (Forman 1995).

Por otro lado, la diversidad de las comunidades vegetales es uno de principales componentes de la biodiversidad que deben ser conservados, ya sea a través de su representación en un sistema de áreas silvestres protegidas, o mediante estrategias de manejo que estén acordes con las características propias de ésta (Noss 1990). En este sentido, la identificación y descripción de unidades vegetacionales, y la incorporación de un mayor número de éstas en un sistema de áreas protegidas, permitirían representar una mayor diversidad de flora y hábitat para otro tipo de organismos, y con ello mejorar la conservación de la biodiversidad (Noss 1987). Para esto, el primer paso es delimitar y caracterizar las unidades vegetacionales de las áreas silvestres protegidas.

La información respecto de la diversidad vegetacional de Chile, especialmente lo referido a los tipos y características de sus unidades fitosociológicas, comprende trabajos realizados tanto a nivel nacional como más zonales o locales. Sin embargo, el grado de conocimiento varía de acuerdo a la zona geográfica. A parte de los trabajos generales a nivel nacional, tales como Schmithüsen (1956), Oberdorfer (1960) y Gajardo (1994). Trabajos zonales o locales más exhaustivos se encuentran principalmente en la región mediterránea de Chile central (e.g. Villaseñor 1980, San M artín et al. 1984, 1986, 1991), y en la región más templada del sur del país (e.g. Pisano 1977, Villagrán 1980, Ramírez et al. 1983, 1991). Es aún escaso el conocimiento de las unidades vegetacionales fitosociológicas de la zona transicional entre las regiones mediterránea y templada $\left(37^{\circ}\right.$ a $\left.39^{\circ} \mathrm{S}\right)$, especialmente en la zona de la precordillera y cordillera andina de la A raucanía (Hildebrand et al. 1990). Estos sólo comprenden los trabajos de Montaldo (1974) en los bosques de A raucaria y Gajardo (1980) en el interior de la Cordillera de Lonquimay (38³0' S). También Ramírez (1978) hace una descripción de las formaciones vegetacionales más importantes del Parque Nacional Tolhuaca $\left(38^{\circ} \mathrm{S}\right)$.

Por otro lado, el conocimiento de la flora y unidades vegetacionales fitosociológicas presentes en el Sistema Nacional de A reas Silvestres Protegidas del Estado en Chile (SNASPE), es aún escaso. No obstante, la representación de grandes unidades vegetacionales fisionómicas (formaciones vegetacionales) en el SNASPE ha sido evaluada por B enoit (1996) y Luebert \& B ecerra (1998). En consecuencia, es importante aumentar el conocimiento de la diversidad vegetacional dentro de las unidades del SNASPE, Io cual permitiría evaluar la representatividad de éste de la biodiversidad de Chile y además orientar su manejo.

En este trabajo se realiza una caracterización de la diversidad vegetacional de la Reserva $\mathrm{Nacional}$ $M$ alal cahuello, IX Región de Chile. Específicamente se hace una delimitación fitosociológica de unidades vegetacionales, se examina la importancia relativa de la variación topográfica y edáfica de la zona en la formación de éstas, y se describe su estructura de especies y formas de vida. 


\section{MATERIAL Y METODOS}

Area del estudio. El estudio se realizó en la Reserva Nacional de Malalcahuello (RNM), ubicada administrativamente en las comunas de Curacautín y Lonquimay, provincia de Malleco, IX Región de la Araucanía, Chile $\left(38^{\circ} 28^{\prime} \mathrm{S}\right)$ (fig. 1).

Según la Clasificación Bioclimática de Di Castri \& Hajek (1976), esta zona se encuentra en la transición de la Región Mediterránea Perhúmeda a la Región Continental Andina. Las observaciones climatológicas de la estación de L onquimay $\mathrm{FACH}$, ubicada en los $38^{\circ} 26^{\prime} \mathrm{S}, 71^{\circ} 14^{\prime} \mathrm{W}$ a una altura de $900 \mathrm{~m}$ s.n.m, la más cercana al área de estudio, muestran que la precipitación media anual es de $1.915,1 \mathrm{~mm}$, sin meses secos. La temperatura media mensual mínima es de $1,9^{\circ} \mathrm{C}$ en julio y la máxima $14,4^{\circ} \mathrm{C}$ en enero. La mínima humedad relativa es en diciembre con $67 \%$ y la máxima en junio y julio con $98 \%$ (Santibáñez 1993).

La fisiografía de la zona es de tipo montañosa con gran variabilidad topográfica. La mayor parte de las rocas fundamentales del área están constituidas principal mente por granodioritas y volcanitas andesítico-basálticas, modeladas por la actividad glacial (Peralta 1980). Posterior al retiro glacial habrían comenzado a formarse los suelos trumaos actuales de esta zona de los A ndes, desarrollados sobre sedimentos glaciales a partir de depositación volcano-eólica (V eit \& Garleff 1995). Los sustratos superficiales son principalmente de tipo arenoso, compuestos especialmente por cenizas, y de tipo pedregoso, compuesto por escorias y gravas. Sin embargo, esta acción no ha cubierto completamente el relieve de las masas rocosas, por lo que gran



Figura 1. Ubicación geográfica de la Reserva Nacional Malalcahuello en la IX Región de Chile. Geographic location of $M$ alalcahuello $N$ ational Reserve in the I $X^{\text {th }}$ Region of Chile. 
parte de la superficie del lugar está configurada por sustratos rocosos. Los sectores cubiertos con mayor biomasa vegetacional han llegado a formar sustratos orgánicos y terrosos. En sectores con reducida profundidad del suelo pueden presentarse sustratos anegados debido a la disminución de la infiltración (Peralta 1980).

La flora de esta zona de Chile y en general del sector preandino y andino del centro-sur de Chile corresponde a aquella resultante de la dispersión postglacial de especies refugiadas en el valle longitudinal y faldas de la Cordillera de la Costa (Villagrán \& Hinojosa 1997) o de migraciones norte-sur a través del cordón andino (Villagrán et al. 1983). La distribución y superficie vegetacional de esta zona ha sido influenciada durante todo el Holoceno por la actividad volcánica, tal como en toda la región centro-sur de Chile (Veblen et al. 1995). Finalmente, la acción antrópica ha sido un importante factor causal del estado del ambiente actual de esta zona de Chile, principalmente a partir de la colonización europea a comienzos del siglo $X I X$. En especial, la tala de bosques para cultivos, el pastoreo, la extracción de leña, y el considerable aumento de incendios, han modificado notablemente el paisaje vegetacional (Ramírez et al. 1988, Lara et al. 1995). La flora de esta Reserva fue caracterizada por Becerra \& Faúndez (1999).

Método de muestreo. El muestreo contempló 100 parcelas denominadas "censos" (MullerDombois \& Ellenberg 1974), en los cuales se registraron las especies vegetales vasculares presentes y su cobertura según las categorías de BraunBlanquet (1950) $(r,+, 1,2,3,4,5)$. L as especies que integraron cada censo corresponden a aquellas que se presentaron en una superficie mayor al área mínima. Para esto se emplearon las siguientes superficies: $4 \mathrm{~m}^{2}, 16 \mathrm{~m}^{2}, 36 \mathrm{~m}^{2}, 64 \mathrm{~m}^{2}, 100 \mathrm{~m}^{2} \mathrm{y}$ $144 \mathrm{~m}^{2}$. Todos los censos presentaron un área mínima menor o igual a $100 \mathrm{~m}^{2}$, por lo cual esta última fue utilizada como superficie de cada censo. Los censos se distribuyeron en transectas altitudinales aproximadamente cada $100 \mathrm{~m}$ de altitud, abarcando todas las exposiciones posibles y en todos los sectores de la reserva, con el fin de captar toda la variabilidad ambiental y florística del área y de cada unidad. No obstante, en cada nivel altitudinal, la ubicación exacta de cada censo fue de tipo dirigida, en un sector de fisonomía y composición florística homogénea, con el fin de evitar censos ecotonales.
Para calcular la cobertura de especies y formas de vida por unidad vegetacional, los valores de abundancia mencionados se transformaron de la siguiente manera: " $r$ " $=0,1 \%, "{ }^{\prime \prime}=1 \%, " 1 "=3 \%$, "2" = 15\%, " 3" = 38\%, "4" = 63\%, y " $5 "=88 \%$. Estos porcentajes corresponden a la proporción del área de cada censo (cobertura absoluta). La cobertura relativa de cada especie por unidad se obtuvo sumando la cobertura de los censos de la unidad, estandarizando entre 0 y 100.

Método de delimitación de unidades vegetacionales. La diferenciación de unidades de vegetación se realizó a través de la metodología fitosociológica de tabulación manual de agrupación y diferenciación de censos, siguiendo los pasos planteados por M uller-Dombois \& Ellenberg (1974) y Ramírez \& W estermeier (1976). Es decir, a partir de una tabla fitosociológica inicial se determinaron especies diferenciales (aquellas que se presentan en los mismos censos y que se excluyen con otras). Los conjuntos de censos en que se presentaron las mismas especies diferenciales correspondieron a las unidades fitosociológicas.

Distribución y segregación ambiental de las unidades vegetacionales. $\mathrm{L}$ a distribución ambiental de cada unidad se estableció a través de la amplitud o número total de posiciones altitudinales, de exposición y tipos de sustratos en que se presentan, los cuales fueron registrados en cada censo. La exposición incluyó todas las posibles exposiciones más la condición de sectores planos y de exposición indefinida (fondos de quebradas). El tipo de sustrato se clasificó en arenoso, pedregoso, rocoso, terroso, orgánico y acuoso. La importancia de un factor ambiental en la formación de las diferentes unidades vegetacionales se analizó a través del nivel de segregación que las unidades presentaron respecto a todas las situaciones o estados posibles de cada factor ambiental (W hittaker 1973). Esto se evaluó, para cada factor, por medio del porcentaje de sobreposición de unidades, es decir, a través del promedio de la proporción de unidades por cada estado de cada variable ambiental. Los valores de sobreposición promedio varían entre 0 y 100. Un menor valor de sobreposición indicaría una alta segregación y un mayor efecto del factor en determinar la formación de las unidades vegetacionales. Un valor de sobreposición promedio igual a 100, para un factor determinado, indicaría que todas las unidades se presentan en todos 
los estados del factor, no existiendo segregación. Por esto, se evaluó si para un factor la segregación es estadísticamente significativa comparando el valor de sobreposición con 100. Luego, con el fin de establecer si existen diferencias del promedio de sobreposición entre los factores, éstos se compararon con pruebas de medias.

Estructura de especies. La estructura de especies de las unidades vegetacionales se evaluó en cuanto a los patrones de diversidad de especies y de orígenes fitogeográficos de las especies. La diversidad de especies se evaluó en cuanto a la diversidad alfa y beta (Whittaker 1972). La diversidad alfa correspondió a la riqueza o número de especies de cada unidad vegetacional. La diversidad beta se expresó en la similitud de especies entre las unidades vegetacionales. La similitud de especies se evaluó a través del índice de Jaccard (Muller-Dombois \& Ellenberg 1974), considerando sólo la presencia/ausencia de las especies. En este caso, el índice relaciona el número de especies compartidas con el número total de especies distintas entre dos unidades:

$$
I(A, B)=\frac{a}{b+c}
$$

donde:

a, número de especies compartidas,

b, número de especies exclusivas de la unidad "A ",

c, número de especies exclusivas de la unidad "B",

I, índice de similitud de Jaccard.

Los orígenes fitogeográficos de las especies, es decir su carácter de introducido o autóctono, se estableció según M articorena \& Quezada (1985). En base a esto se evaluó la proporción de especies que son introducidas y autóctonas por unidad vegetacional.

Estructura de formas de vida. En primera instancia se asignó a cada especie una forma de vida en base a las propuestas por Raunkiaer (1937). La estructura de formas de vida de cada unidad se caracterizó por la diversidad de formas de vida en función del número de especies por forma de vida y en función de la abundancia relativa de cada forma de vida. En el primer caso se construyó el espectro de riqueza de especies por forma de vida y luego se calculó la diversidad a través del siguiente índice:

$$
H=-\square\left(p_{i} \ln p_{i}\right)
$$

donde pj es la proporción de especies que presentan la forma de vida i-ésima. $\mathrm{H}$ indica el nivel de dominancia (riqueza relativa) de una o pocas formas de vida. M ientras menor es su valor más dominada es la unidad por pocas formas de vida y por ello menos diversa. Mientras mayor es el valor, menos dominada es la unidad por pocas formas de vida y con ello más diversa (K rebs 1989).

Para el cálculo de la diversidad de formas de vida en función de la abundancia de éstas se evaluó en primera instancia el espectro de cobertura relativa de cada forma de vida, y luego se calculó el mismo índice anterior pero modificando los valores de "pi" por la proporción de cobertura de cada forma de vida. La cobertura relativa de cada forma de vida corresponde a la suma de las coberturas relativas de las especies que conforman cada forma de vida. Además, la cobertura relativa de cada forma de vida permite conocer la fisonomía vegetacional de cada unidad. Finalmente se correlacionaron ambos índices para ver si las unidades con mayor diversidad de formas de vida según riqueza de especies son también más diversas según cobertura.

\section{RESULTADOS}

Unidades vegetacionales fitosociológicas. Se delimitaron 13 unidades fitosociológicas, cuyos nombres y tabla fitosociológica ordenada mostrando sólo las especies diferenciales de la vegetación se presentan en el cuadro 1. Las unidades presentaron distintos números de especies diferenciales. Existen unidades cuyas especies diferenciales se presentaron sólo en ellas, mientras que también hubo otras con especies diferenciales compartidas. Los nombres de las unidades son provisorios y sólo son válidos para su referencia en este trabajo, ya que todas éstas debieran, posteriormente, ser clasificadas dentro de la sintaxonomía fitosociológica de la vegetación de Chile. Los nombres están dados por las dos especies (no necesariamente diferenciales) con mayor frecuencia en la unidad. Si en una unidad se presentaron más dos especies con igual mayor valor de frecuencia, se consideraron entre ellas las dos con mayor cobertura relativa. Se excluyó dentro de las especies del nombre a Chusquea argentina Parodi, ya que en todas las unidades se presentó con una alta frecuencia y co- 
Tabla fitosociológica ordenada de la vegetación de la R N M

Ordered Phytosociological Table of Vegetation of RNM

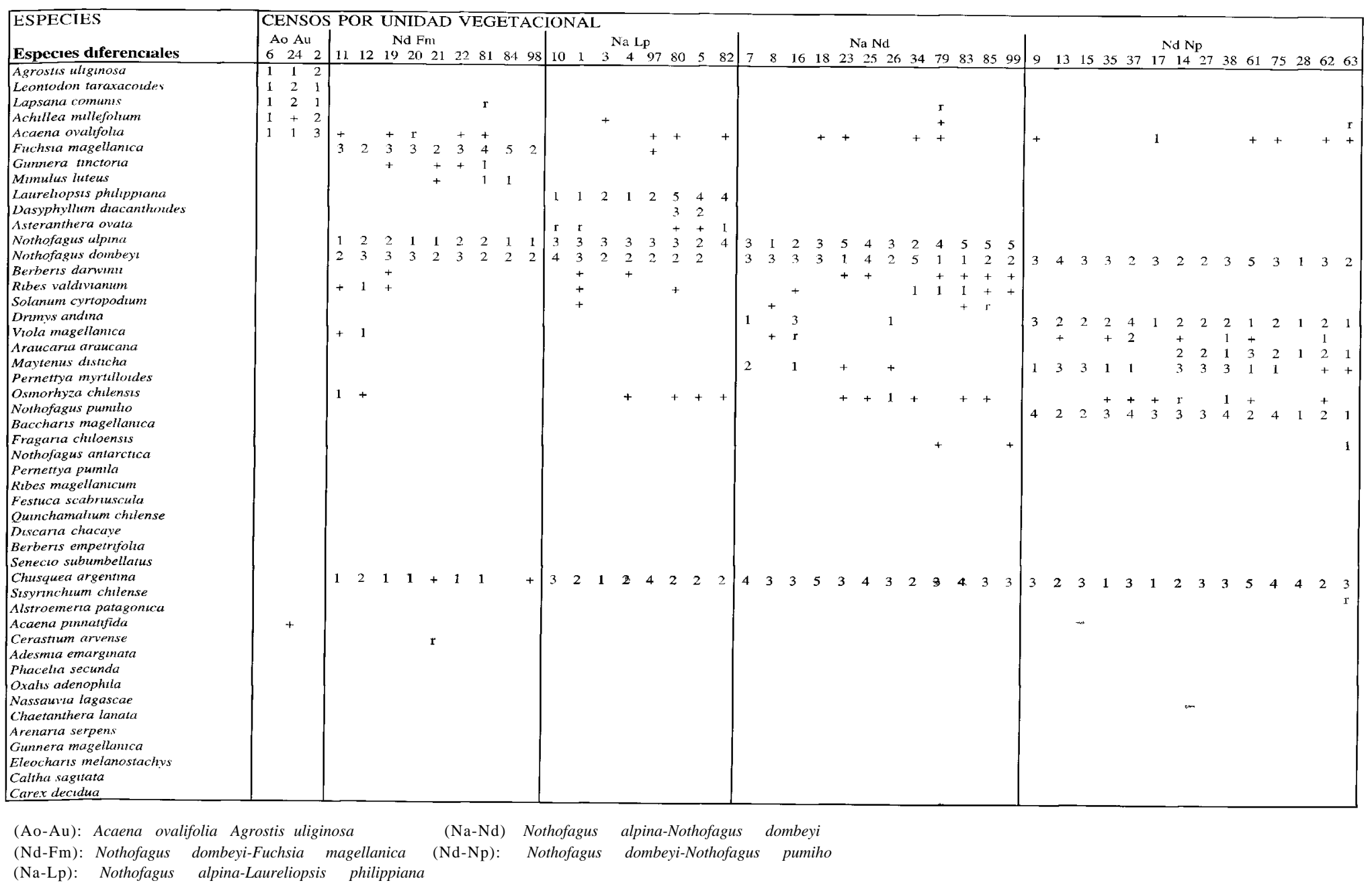




\section{CUADRO 1 (Continuación)}

Tabla fitosociológica ordenada de la vegetación de la RNM. Ordered Phytosociological Table of $V$ egetation of RNM.

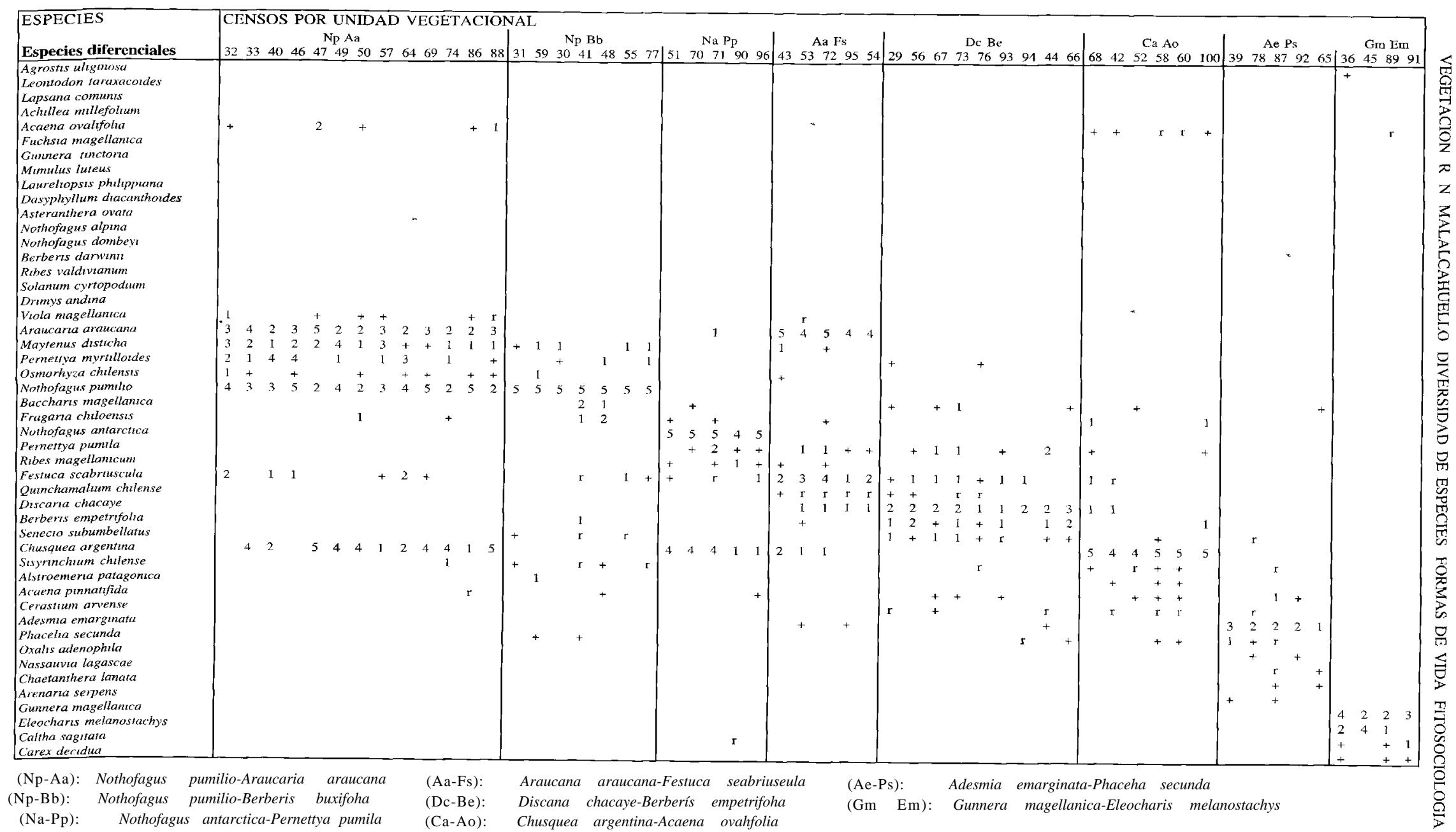


bertura. Esta especie sólo se empleó en el nombre de la unidad donde es la única que posee mayor frecuencia y cobertura.

Distribución ambiental de las unidades vegetacionales. Respecto a la distribución en altitud, las unidades con más amplia distribución (400 m) son $\mathrm{Na}-\mathrm{Nd}$ y $\mathrm{Nd}-\mathrm{Np}$. La unidad Ao-A u es la de menor amplitud altitudinal $(100 \mathrm{~m})$. Las demás poseen en promedio una amplitud de 200 a $300 \mathrm{~m}$ (figura 2A). Las unidades vegetacionales poseen una sobreposición altitudinal promedio de $28,2 \%$, es decir, de las 13 unidades vegetacionales, el $28,2 \%$ como promedio se presenta en cada nivel altitudinal. Este valor difiere significativamente de $100(t=14,9 ; p<0,05)$. La figura $2 \mathrm{~B}$ muestra la distribución de las unidades respecto a un eje ordenado de exposiciones según un posible gradiente de humedad (W hittaker 1973). No se observa una marcada preferencia de unidades por algún extremo del gradiente, sino más bien éstas se presentan indistintamente en los diferentes tipos de exposición. La sobreposición promedio en las distintas exposiciones es de $41,5 \%$. Este valor difiere significativamente de 100 ( $t=16,82 ; p<0,05)$. Finalmente, la figura $2 \mathrm{C}$ muestra la distribución de las unidades en los distintos tipos de sustratos presentes en la RN M. La unidad N d-N p es la de mayor amplitud de sustratos (4). La unidad Gm-Em es la de menor amplitud, presente en sólo un tipo de sustrato, siendo además la única presente en el de tipo acuoso. Cabe mencionar también a la unidad $D c-B e$, única presente en sustratos rocosos, aunque también en sustratos pedregosos. La sobreposición promedio en los tipos de sustratos es de $29,5 \%$. Este valor difiere significativamente de 100 $(t=8,1 ; p<0,05)$. Sólo se encontraron diferencias significativas de los promedios de sobreposición entre la altitud y la exposición (cuadro 2). No existen diferencias entre exposición y sustrato o sustrato $y$ altitud (cuadro 2).

Estructura de especies de las unidades vegetacionales. En cuanto a la diversidad de especies, el número total de especies encontrado en la RNM es 211. La diversidad alfa presenta una amplia variación entre las unidades vegetacionales (cuadro 3). El rango de variación va entre 58 $(27,5 \%)$ y 21 especies $(10 \%)$. Por otro lado, la diversidad beta, o similitud de especies entre unidades, difiere dependiendo del par de unidades comparadas. No obstante, los valores de similitud son en general bajos, con un rango entre 0 y 0,509 (cuadro 4). Es decir, el porcentaje de especies compartidas entre unidades varió desde un $0 \%$ hasta un $50,9 \%$. Los pares de unidades con mayor similitud de especies fueron $\mathrm{Na}-\mathrm{LP}$ y $\mathrm{Na}-\mathrm{Nd}$ $(0,509), \mathrm{N} \mathrm{d}-\mathrm{Fm}$ y $\mathrm{Na}-\mathrm{Nd}(0,406), \mathrm{N} \mathrm{d}-\mathrm{Fm}$ y Na-Lp $(0,417)$ y N d-N p y N -Aa $(0,410)$. Las unidades vegetacionales que no compartieron especies (similitud de 0\%) son A o-Au con Aa-Fs y A e-Ps con Gm-Em (cuadro 4). Los promedios de similitudes por unidad varían entre 0,042 y 0,166 (cuadro 4). La unidad que posee menos especies compartidas en promedio ( $4,2 \%$ de las especies) es Gm-Em, y la de mayor semejanza promedio $(16,6 \%$ de las especies) con otras es la unidad Np-A a. La composición de cada unidad vegetacional se presenta en el A péndice 1.

En cuanto al origen fitogeográfico de las especies, el $10,9 \%$ de las especies de la reserva son introducidas. La invasión de especies introducidas en cada unidad vegetacional es en general baja (cuadro 5). Sólo una unidad presentó un porcentaje considerablemente alto de especies introducidas (unidad Ao-A u con 56\%). Sólo dos unidades no presentaron especies introducidas (unidades $\mathrm{Na}$-Pp y Aa-Fs). El origen fitogeográfico de cada especie se presenta en el A péndice 1.

Estructura de formas de vida de las unidades vegetacionales. La forma de vida y abundancia de las especies se presenta en el A péndice 1 . En cuanto a la diversidad de formas de vida según la riqueza de especies, cabe destacar que en ninguna unidad se presentan todas las formas de vida y que en todas las unidades sólo son una o dos las formas de vida que poseen una alta riqueza relativa de especies (cuadro 6). Entre los resultados más sobresalientes puede mencionarse la gran riqueza relativa de hemicriptófitas (56\%) de la unidad A o-A u y de geófitas $(54,5 \%)$ en la unidad G m-E m. Las macrofanerófitas y nanofanerófitas poseen su mayor importancia en la unidad Na-L $p$. Las lianas fanerófitas alcanzan su mayor importancia en las unidades $\mathrm{Na}-\mathrm{Lp}$ y Nd-Np. Por su parte las especies caméfitas presentan su mayor riqueza relativa en la unidad N p-Bb. Finalmente, las terófitas presentan su mayor importancia en la unidad $\mathrm{Nd}-\mathrm{Fm}$ y las parásitas en la unidad N P-A a. Todo lo anterior determina que la diversidad de formas de vida varíe entre las unidades. La unidad más diversa es $\mathrm{Nd}$ Fm $(1,68)$ y la menos diversa G m-E m $(1,05)$ (cuadro 6). La heterogeneidad media es de 1,47. 
A)

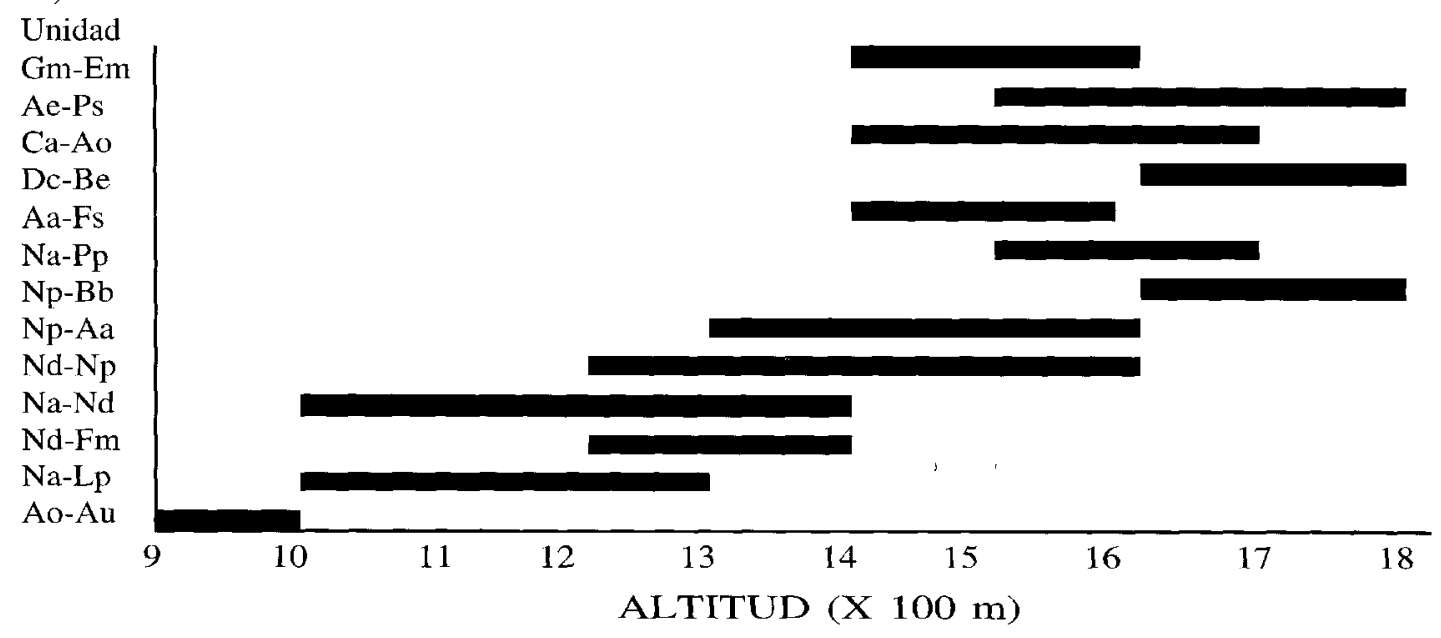

B)

Unidad Gm-Em Ae-Ps $\mathrm{Ca}-\mathrm{Ao}$ $\mathrm{Dc}-\mathrm{Be}$ Aa-Fs Na-Pp $\mathrm{Np}-\mathrm{Bb}$ Np-Aa Nd-Np $\mathrm{Na}-\mathrm{Nd}$ Nd-Fm Na-Lp $\mathrm{Ao-Au}$

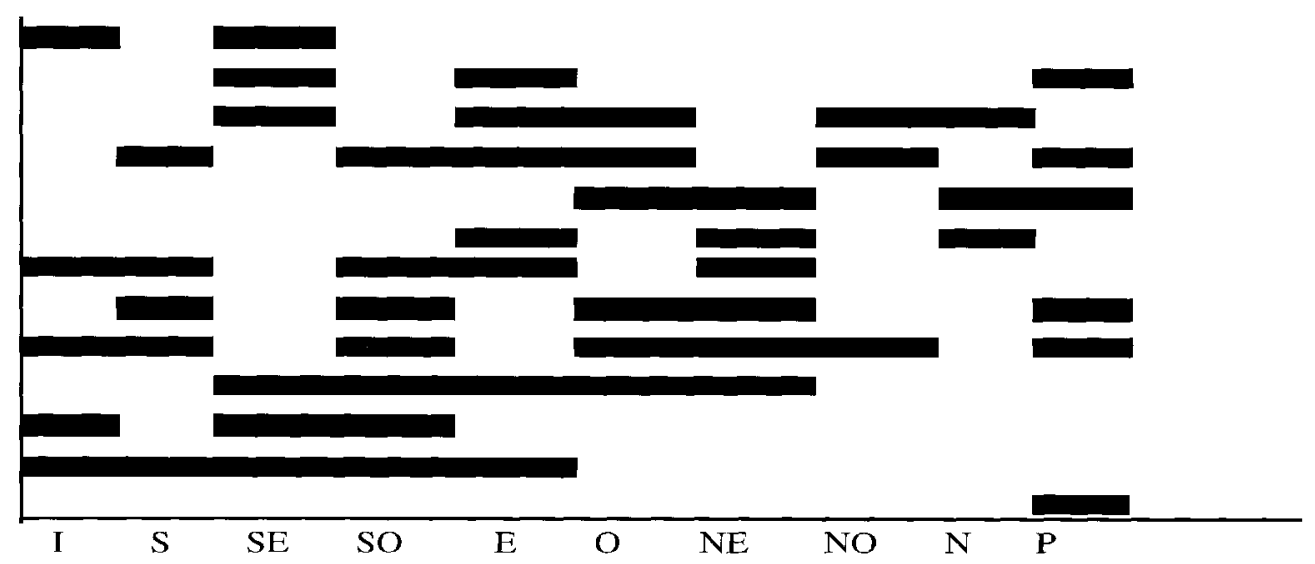

C) EXPOSICION (I: indefinido; P: plano)

Unidad Gm-Em Ae-Ps Ca-Ao $\mathrm{Dc}-\mathrm{Be}$ Aa-Fs $\mathrm{Na}-\mathrm{Pp}$ $\mathrm{Np}-\mathrm{Bb}$ $\mathrm{Np}-\mathrm{Aa}$ $\mathrm{Nd}-\mathrm{Np}$ $\mathrm{Na}-\mathrm{Nd}$ $\mathrm{Nd}-\mathrm{Fm}$ Na-Lp Ao-Au

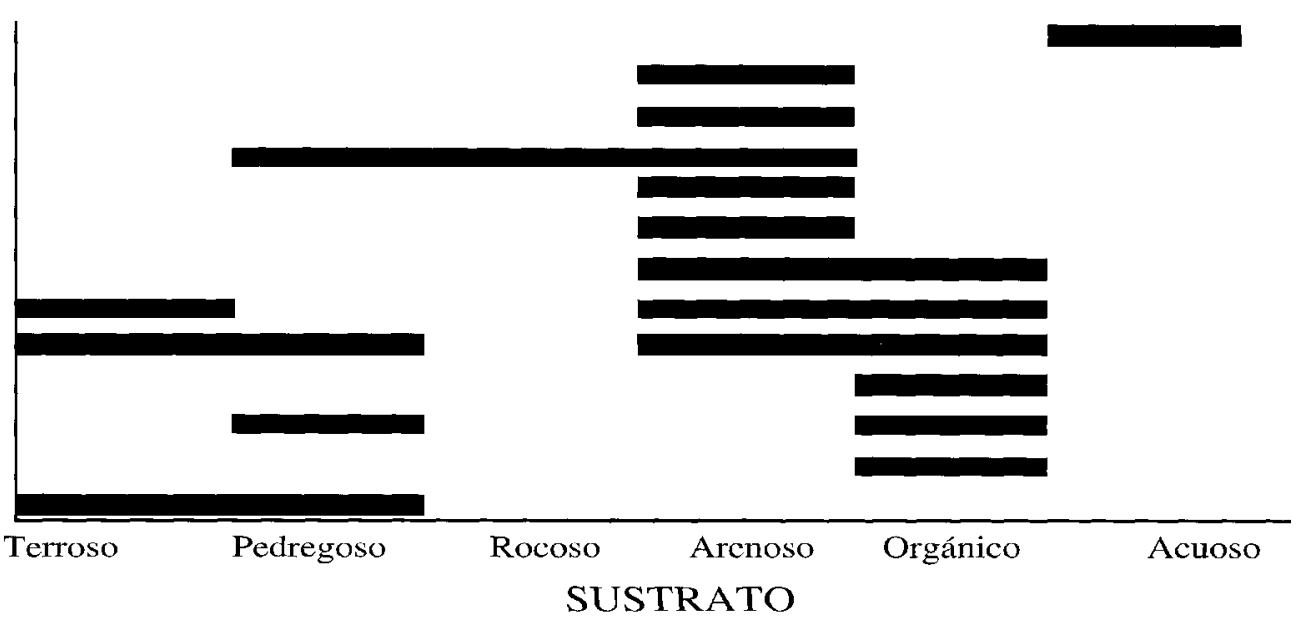

Figura 2. Distribución de las unidades vegetacionales en altitud (A), exposición (B) y sustrato (C). Distxibution of vegetation units in elevation (A), exposition (B) and substrate (C). 


\section{CUADRO 2}

Valores de $t$-student para las comparaciones de segregación de unidades entre factores ambientales.

Student $t$ values for comparisons of segregation of units between environmental factors.

\begin{tabular}{|ccc|}
\hline FACTOR & Altitud & Sustrato \\
\hline Exposición & $t=2,278^{*}$ & $t=1,506$ \\
Sustrato & $t=0,131$ & \\
\hline
\end{tabular}

Valor estadísticamente significativo con $p<0,05$

\section{CUADRO 3}

Riqueza de especies absoluta y relativa de las unidades vegetacionales de la RNM.

A bsolute and relative species richness of vegetation units of RNM

\begin{tabular}{|c|c|c|}
\hline $\begin{array}{c}\text { Unidad } \\
\text { V egetacional }\end{array}$ & $\begin{array}{c}\text { Riqueza total } \\
N^{\circ} \text { Esp. }\end{array}$ & $\begin{array}{l}\text { Riqueza relativa } \\
\% \text { de flora total }\end{array}$ \\
\hline $\mathrm{A} O-\mathrm{A} u$ & 25 & 11,8 \\
\hline $\mathrm{Nd}-\mathrm{Fm}$ & 48 & 22,8 \\
\hline $\mathrm{Na}-\mathrm{Lp}$ & 37 & 17,5 \\
\hline $\mathrm{Na}-\mathrm{Nd}$ & 49 & 23,2 \\
\hline $\mathrm{Nd}-\mathrm{Np}$ & 49 & 23,2 \\
\hline $\mathrm{Np}-\mathrm{Aa}$ & 44 & 20,9 \\
\hline$N p-B b$ & 38 & 18,0 \\
\hline $\mathrm{Na}-\mathrm{Pp}$ & 21 & 10,0 \\
\hline $\mathrm{Aa}-\mathrm{Fs}$ & 34 & 16,1 \\
\hline$D C-B e$ & 58 & 27,5 \\
\hline $\mathrm{Ca}-\mathrm{Ao}$ & 43 & 20,4 \\
\hline $\mathrm{A}$ e-Ps & 22 & 10,4 \\
\hline Gm-Em & 22 & 10,4 \\
\hline
\end{tabular}


CUADRO 4

Matriz de similitud de especies entre las unidades de la RNM.

Matrix of species similanties between units of RNM.

\begin{tabular}{|c|c|c|c|c|c|c|c|c|c|c|c|c|c|}
\hline Unidad & Na-Lp & Nd-Fm & $\mathrm{Na}-\mathrm{Nd}$ & Nd-Np & $\mathrm{Np}-\mathrm{Aa}$ & $\mathrm{Np}-\mathrm{Bb}$ & $\mathrm{Na}-\mathrm{Pp}$ & $\mathrm{Aa}-\mathrm{Fs}$ & Dc-Be & Ca-Ao & $\mathrm{Ae}-\mathrm{Ps}$ & $\mathrm{Gm}-\mathrm{Em}$ & Promedio \\
\hline Ao-Au & 0,148 & 0,140 & 0,156 & 0,057 & 0,062 & 0,033 & 0,022 & 0,000 & 0,025 & 0,046 & 0,044 & 0,044 & 0,065 \\
\hline $\mathrm{Na}-\mathrm{Lp}$ & 1,000 & 0,417 & 0,509 & 0,284 & 0,157 & 0,056 & 0,018 & 0,060 & 0,011 & 0,039 & 0,017 & 0,035 & 0,146 \\
\hline $\mathrm{Nd}-\mathrm{Fm}$ & & 1,000 & 0,406 & 0,228 & 0,150 & 0,036 & 0,015 & 0,051 & 0,010 & 0,058 & 0,029 & 0,045 & 0,132 \\
\hline $\mathrm{Na}-\mathrm{Nd}$ & & & 1,000 & 0,342 & 0,192 & 0,074 & 0,029 & 0,078 & 0,019 & 0,057 & 0,014 & 0,029 & 0,158 \\
\hline $\mathrm{Nd}-\mathrm{Np}$ & & & & 1,000 & 0,410 & 0,145 & 0,077 & 0,153 & 0,059 & 0,095 & 0,044 & 0,060 & 0,163 \\
\hline $\mathrm{Np}-\mathrm{Aa}$ & & & & & 1,000 & 0,302 & 0,182 & 0,182 & 0,097 & 0,130 & 0,048 & 0,082 & 0,166 \\
\hline $\mathrm{Np}-\mathrm{Bb}$ & & & & & & 1,000 & 0,157 & 0,200 & 0,247 & 0,227 & 0,132 & 0,034 & 0,137 \\
\hline $\mathrm{Na}-\mathrm{Pp}$ & & & & & & & 1,000 & 0,222 & 0,113 & 0,164 & 0,049 & 0,075 & 0,093 \\
\hline Aa-Fs & & & & & & & & 1,000 & 0,226 & 0,203 & 0,037 & 0,018 & 0,119 \\
\hline $\mathrm{Dc}-\mathrm{Be}$ & & & & & & & & & 1,000 & 0,278 & 0,250 & 0,013 & 0,112 \\
\hline $\mathrm{Ca}-\mathrm{Ao}$ & & & & & & & & & & 1,000 & 0,182 & 0,066 & 0,128 \\
\hline $\mathrm{Ae}-\mathrm{Ps}$ & & & & & & & & & & & 1,000 & 0,000 & 0,071 \\
\hline Gm-Em & & & & & & & & & & & & 1,000 & 0,042 \\
\hline
\end{tabular}

CUADRO 5

Proporción de especies introducidas y autóctonas por unidad vegetacional.

Percentage of introduced and native species per vegetation unit.

\begin{tabular}{|lrrrr|}
\hline \multirow{2}{*}{ Unidad vegetacional } & \multicolumn{2}{c}{ Autóctonas } & \multicolumn{2}{c|}{ Introducidas } \\
\cline { 2 - 5 } & \multicolumn{1}{c}{ № } & $\%$ & № & $\%$ \\
\hline Aa-Au & 11 & 44,0 & 14 & 56,0 \\
Na-Lp & 31 & 83,8 & 6 & 16,2 \\
Nd-Fm & 36 & 75,0 & 12 & 25,0 \\
Na-Nd & 38 & 77,6 & 11 & 22,4 \\
Nd-Np & 47 & 95,9 & 2 & 4,1 \\
Aa-Np & 43 & 97,7 & 1 & 2,3 \\
Np-Bb & 36 & 94,7 & 2 & 5,3 \\
Na-Pp & 21 & 100,0 & 0 & 0,0 \\
Aa-Fs & 34 & 100,0 & 0 & 0,0 \\
Dc-Be & 56 & 96,6 & 2 & 3,4 \\
Ca-Ao & 39 & 90,7 & 4 & 9,3 \\
Ae-Ps & 20 & 90,9 & 2 & 9,1 \\
Gm-Em & 21 & 95,5 & 1 & 4,5 \\
\hline
\end{tabular}


CUADRO 6

Riqueza absoluta y relativa de especies, y diversidad de formas de vida por unidad vegetacional. Relative and absolute species richness and diversity of life forms per vegetational unit.

\begin{tabular}{|c|c|c|c|c|c|c|c|c|c|c|}
\hline \multirow{2}{*}{$\begin{array}{l}\text { Unidad } \\
\text { vegetacional }\end{array}$} & \multicolumn{9}{|c|}{ Formas de vida } & \multirow{2}{*}{$\begin{array}{c}\text { Diversidad } \\
\text { (H) }\end{array}$} \\
\hline & & $\mathrm{M}$ & $\mathrm{N}$ & $\mathrm{L}$ & C & $\mathrm{H}$ & $\mathrm{G}$ & $\mathrm{T}$ & $\mathrm{P}$ & \\
\hline \multirow[t]{2}{*}{$\mathrm{Ao}-\mathrm{Au}$} & $\mathrm{N}^{\circ}$ & 1 & 3 & 0 & 4 & 14 & 1 & 2 & 0 & 1,33 \\
\hline & $\%$ & 4 & 12 & 0 & 16 & 56 & 4 & 8 & 0 & \\
\hline \multirow[t]{2}{*}{$\mathrm{Na}-\mathrm{Lp}$} & $\mathrm{N}^{\circ}$ & 5 & 13 & 3 & 2 & 12 & 2 & 0 & 0 & 1,52 \\
\hline & $\%$ & 13,5 & 35,1 & 8,1 & 5,4 & 32,4 & 5,4 & 0 & 0 & \\
\hline \multirow[t]{2}{*}{ Nd-Fm } & $\mathrm{N}^{\circ}$ & 3 & 11 & 2 & 2 & 17 & 8 & 5 & 0 & 1,68 \\
\hline & $\%$ & 6,3 & 22,9 & 4,2 & 4,2 & 35,4 & 16,7 & 10,4 & 0 & \\
\hline \multirow[t]{2}{*}{$\mathrm{Na}-\mathrm{Nd}$} & $\mathrm{N}^{\circ}$ & 4 & 17 & 2 & 2 & 16 & 6 & 2 & 0 & 1,59 \\
\hline & $\%$ & 8,2 & 34,7 & 4,1 & 4,1 & 32,7 & 12,2 & 4,1 & 0 & \\
\hline \multirow[t]{2}{*}{$\mathrm{Nd}-\mathrm{Np}$} & $\mathrm{N}^{\mathrm{o}}$ & 3 & 11 & 4 & 2 & 15 & 13 & 0 & 1 & 1,63 \\
\hline & $\%$ & 6,1 & 22,4 & 8,2 & 4,1 & 30,6 & 26,5 & 0 & 2 & \\
\hline \multirow[t]{2}{*}{$\mathrm{Np}-\mathrm{Aa}$} & $\mathbf{N}^{0}$ & 2 & 10 & 2 & 5 & 9 & 15 & 0 & 1 & 1,64 \\
\hline & $\%$ & 4,5 & 22,7 & 4,5 & 11,4 & 20,5 & 34,1 & 0 & 2,3 & \\
\hline \multirow[t]{2}{*}{$\mathrm{Np}-\mathrm{Bb}$} & $\mathrm{N}^{\mathrm{o}}$ & 1 & 6 & 1 & 13 & 8 & 9 & 0 & 0 & 1,52 \\
\hline & $\%$ & 2,6 & 15,8 & 2,6 & 34,2 & 21,1 & 23,7 & 0 & 0 & \\
\hline \multirow[t]{2}{*}{ Na-Pp } & $\mathrm{N}^{\mathrm{o}}$ & 2 & 4 & 0 & 5 & 3 & 7 & 0 & 0 & 1,53 \\
\hline & $\%$ & 9,5 & 19 & 0 & 23,8 & 14,3 & 33,3 & 0 & 0 & \\
\hline \multirow[t]{2}{*}{ Aa-Fs } & $\mathrm{N}^{\circ}$ & 1 & 8 & 1 & 8 & 11 & 5 & 0 & 0 & 1,53 \\
\hline & $\%$ & 2,9 & 23,5 & 2,9 & 23,5 & 32,4 & 14,7 & 0 & 0 & \\
\hline \multirow[t]{2}{*}{$\mathrm{Dc}-\mathrm{Be}$} & $\mathrm{N}^{\circ}$ & 0 & 8 & 1 & 19 & 19 & 11 & 0 & 0 & 1,39 \\
\hline & $\%$ & 0 & 13,8 & 1,7 & 32,8 & 32,8 & 19 & 0 & 0 & \\
\hline \multirow[t]{2}{*}{$\mathrm{Ca}-\mathrm{Ao}$} & $\mathrm{N}^{\circ}$ & 0 & 4 & 1 & 13 & 14 & 10 & 1 & 0 & 1,46 \\
\hline & $\%$ & 0 & 9,3 & 2,3 & 30,2 & 32,6 & 23,3 & 2,3 & 0 & \\
\hline \multirow[t]{2}{*}{ Ae-Ps } & $\mathrm{N}^{\mathrm{o}}$ & 0 & 1 & 0 & 6 & 9 & 6 & 0 & 0 & 1,21 \\
\hline & $\%$ & 0 & 4,5 & 0 & 27,3 & 40,9 & 27,3 & 0 & 0 & \\
\hline \multirow[t]{2}{*}{$\mathrm{Gm}-\mathrm{Em}$} & $\mathrm{N}^{\circ}$ & 0 & 1 & 0 & 2 & 7 & 12 & 0 & 0 & 1,05 \\
\hline & $\%$ & 0 & 4,5 & 0 & 9,1 & 31,8 & 54,5 & 0 & 0 & \\
\hline
\end{tabular}
M: M acrofanerófita
H: Hemicriptófita
$N$ : Nanofanerófita
G: Geófita
L: Liana fanerófita
$\mathrm{T}$ : Terófita
C: Caméfita
P: Parásita 


\section{CUADRO 7}

Cobertura relativa (\%) y diversidad de formas de vida según cobertura por unidad vegetacional. Relative coverage (\%) and diversity of life forms per vegetation unit.

\begin{tabular}{|c|c|c|c|c|c|c|c|c|c|}
\hline \multirow{2}{*}{$\begin{array}{l}\text { Unidad } \\
\text { vegetacional }\end{array}$} & \multicolumn{8}{|c|}{ Formas de vida } & \multirow{2}{*}{$\begin{array}{c}\text { Diversidad } \\
(H)\end{array}$} \\
\hline & M & $\mathrm{N}$ & $\mathrm{L}$ & C & $\mathrm{H}$ & G & T & $P$ & \\
\hline A o-A u & 12,4 & 6,1 & 0,0 & 18,6 & 44,7 & 5,8 & 12,3 & 0,0 & 1,53 \\
\hline $\mathrm{Na}-\mathrm{Lp}$ & 73,4 & 22,9 & 0,5 & 0,4 & 1,8 & 0,9 & 0,0 & 0,0 & 0,70 \\
\hline $\mathrm{Nd}-\mathrm{Fm}$ & 37,5 & 52,7 & 0,2 & 0,7 & 2,7 & 4,8 & 1,5 & 0,0 & 1,04 \\
\hline $\mathrm{Na}-\mathrm{Nd}$ & 57,3 & 40,4 & 0,2 & 0,4 & 1,4 & 0,4 & 0,0 & 0,0 & 0,79 \\
\hline $\mathrm{Nd}-\mathrm{Np}$ & 49,7 & 45,9 & 0,4 & 0,5 & 2,6 & 0,8 & 0,0 & 0,1 & 0,87 \\
\hline $\mathrm{Np}-\mathrm{A} a$ & 47,5 & 44,2 & 0,2 & 1,2 & 3,5 & 3,0 & 0,0 & 0,3 & 0,99 \\
\hline$N p-B b$ & 80,8 & 5,0 & 0,1 & 7,8 & 3,9 & 2,3 & 0,0 & 0,0 & 0,73 \\
\hline $\mathrm{Na}-\mathrm{Pp}$ & 83,2 & 2,4 & 0,0 & 5,6 & 0,3 & 8,5 & 0,0 & 0,0 & 0,63 \\
\hline $\mathrm{Aa}-\mathrm{Fs}$ & 62,3 & 6,9 & 0,3 & 5,5 & 1,2 & 23,7 & 0,0 & 0,0 & 1,03 \\
\hline$D C-B e$ & 0,0 & 7,6 & 0,5 & 70,1 & 13,9 & 7,8 & 0,0 & 0,0 & 0,92 \\
\hline Ca-Ao & 0,0 & 87,5 & 0,7 & 6,2 & 2,0 & 3,5 & 0,0 & 0,0 & 0,49 \\
\hline A e-Ps & 0,0 & 4,9 & 0,0 & 77,4 & 11,8 & 5,9 & 0,0 & 0,0 & 0,76 \\
\hline Gm-Em & 0,0 & 0,4 & 0,0 & 0,9 & 56,1 & 42,6 & 0,0 & 0,0 & 0,75 \\
\hline
\end{tabular}

$\begin{array}{lc}\text { M: Macrofanerófíta } & H: \text { Hemicriptófita } \\ \text { N: Nanofanerófita } & \text { G: Geófita } \\ \text { L: Liana fanerófita } & \text { T: Terófita } \\ \text { C: Caméfita } & \text { P: Parásita }\end{array}$

Al evaluar la diversidad de formas de vida en función de la cobertura de éstas, puede observarse que todas las unidades presentan una o dos formas de vida dominantes, mientras que las demás se encuentran poco abundantes 0 ausentes (cuadro 7). L as formas de vida constantemente menos abundantes son la terófita y parásita, mientras que las más abundantes son las macro y nanofanerófitas. Cabe resaltar la gran dominancia de macrofanerófitas en las unidades $\mathrm{Na}$-Pp con el $83,2 \%$ de la cobertura y $\mathrm{Np}-\mathrm{Bb}$ con el $80,8 \%$ de la cobertu$\mathrm{ra}$, y de nanofanerófitas en la unidad $\mathrm{Ca}$-A $\mathrm{o}$ con el $87,5 \%$. Lo anterior determina que exista un amplio rango de valores de diversidad de formas de vida según la cobertura de éstas (cuadro 7). La unidad más diversa en este caso es Ao-A u, con un índice de 1,5 , mientras que la unidad menos diversa es $\mathrm{Ca}-\mathrm{A} \mathrm{O}$, con un índice de 0,49 . El promedio de los valores de diversidad de formas de vida según cobertura es 0,87 .

Los valores de diversidad de formas de vida según número de especies y cobertura por forma de vida no están correlacionados entre las unidades $(r=0,024 ; p=0,937)$. Es decir, las unidades más diversas al considerar el número de especies por forma de vida no son las más diversas según la cobertura por forma de vida.

\section{DISCUSION Y CONCLUSIONES}

La vegetación de la Reserva Nacional $M$ alalcahuello posee una diversidad de 13 unidades vegetacionales fitosociológicas, cuya identidad y jerarquía sintaxonómica debe ser analizada posteriormente. No obstante, debido al bajo número de censos con que éstas se definieron, para su posterior clasificación sintaxonómica debiera muestrearse un mayor número de censos por unidad alcanzando al menos a 10 (Barkman et al. 1986).

Si bien los tres factores ambientales relacionados con las unidades vegetacionales generan una segregación significativa de éstas, influyendo así 
en su formación, el más importante es la altitud. Landrum \& Nimios (1975) también encontraron una marcada segregación altitudinal de las principales especies leñosas de la Reserva Nacional $M$ alalcahuello. La gran importancia de la altitud en formar diferentes unidades vegetacionales ya ha sido documentada por Whittaker (1973), Stephenson (1990) y W esser \& A rmbruster (1991), entre otros. Respecto a la exposición, en este trabajo si bien este factor genera una segregación significativa de unidades vegetacionales, es el factor con mayor valor de sobreposición y por lo tanto el menos importante en la formación de éstas. Las diferencias significativas entre el efecto de la altitud y la exposición indican que el primero es significativamente más importante que el segundo. En latitudes más mediterráneas A rmesto et al. (1979) también encuentran un significativo efecto de la exposición sobre la composición de la vegetación; sin embargo, no se conoce su importancia relativa respecto al efecto de la altitud en esta zona de Chile. Finalmente, la significativa segregación de unidades vegetacionales en los distintos tipos de sustratos evidencia que este factor puede llegar a ser determinante en la formación de ciertas unidades vegetacionales particulares, lo cual es también sugerido por Sollins (1998) en bosques tropicales. En la RNM la segregación entre sustratos está dada especialmente por la alta dependencia de la unidad $\mathrm{G} \mathrm{m}$ - $\mathrm{Em}$ por sustratos acuosos y de la unidad DC-Be por sustratos rocosos. $L$ as demás unidades se presentan en general en los mismos tipos de sustratos (arenosos y orgánicos). La exclusiva presencia de algunas unidades vegetacionales en sustratos acuosos es un patrón encontrado a lo largo de toda la Cordillera de los Andes, en la cual existen comunidades vegetales azonales, dependientes exclusivamente de las condiciones anegadas del suelo (Villagrán et al. 1983).

En cuanto a la estructura de especies y formas de vida de las unidades vegetacionales, éstas presentan las siguientes características más importantes:

La unidad Ao-A u posee 25 especies, lo cual implica el $11,8 \%$ de la flora total. Esta unidad también posee uno de los valores de similitud promedio de especies más bajos, lo cual se debería a su composición dominada por especies introducidas, las cuales, en general, aún no han invadido el resto de las unidades vegetacionales. Presenta una baja diversidad de formas de vida al considerar el número de especies, pero es la de mayor diversi- dad de formas de vida al considerar la cobertura de éstas. El primer hecho se debería a la alta proporción de especies hemicriptófitas respecto de las demás formas de vida. El segundo patrón estaría dado por una cobertura más homogénea de las formas de vida; no obstante, existe una mayor dominancia de hemicriptófitas, aunque también se presenta un estrato arbóreo con individuos aislados de Nothofagus obliqua (M irb.) Oerst. y con menor abundancia uno arbustivo. Por esto, su fisonomía es de tipo Parque. Esto, en conjunto con la alta proporción de especies introducidas, la más alta de las unidades, indica su carácter de comunidad secundaria, resultante de la eliminación del bosque de Nothofagus obliqua primitivo.

La unidad $\mathrm{Nd}-\mathrm{Fm}$ posee una riqueza de 48 especies, lo que corresponde al $22,8 \%$ de la flora de la RNM, de las cuales el $25 \%$ son introducidas, siendo la segunda más invadida de la reserva. Es la unidad de mayor diversidad de formas de vida al considerar el número de especies. Esto se debería a que es una de las unidades con mayor número de formas de vida diferentes, y que éstas poseen similares proporciones de especies. Su fisonomía es de un bosque multiestratificado, con una también alta diversidad de formas de vida según la cobertura.

$\mathrm{L}$ a unidad $\mathrm{Na}$ - L p posee una riqueza de 37 especies $(17,5 \%)$, de las cuales el $16,2 \%$ son introducidas. Posee una alta diversidad de especies por forma de vida, pero con una baja diversidad de formas de vida según cobertura, debido a la alta dominancia de macrofanerófitas y nanofanerófitas. Su fisonomía es de bosque multiestratificado. Ramírez \& Figueroa (1987) y Pollmann (2000) también han definido como unidad fitosociológica a sitios dominados por $N$. dombeyi, $N$. alpina, $L$. philippiana y $D$. diacanthoides.

$\mathrm{L}$ a unidad $\mathrm{Na}-\mathrm{N}$ d posee una riqueza de 49 especies $(23,2 \%)$, situándose como una de las más ricas de la RNM; no obstante un alto porcentaje corresponde a especies introducidas $(22,5 \%)$. Presenta una alta diversidad de especies por formas de vida, pero con una baja diversidad de formas de vida según la cobertura de éstas, debido a la alta dominancia de macrofanerófitas y nanofanerófitas. Su fisonomía es de bosque multiestratificado.

La unidad $\mathrm{Nd}-\mathrm{NP}$ posee una de las más altas riquezas de especies $(49,23,2 \%)$, y también uno de los menores porcentajes de especies introducidas. Posee una alta diversidad de especies por for- 
ma de vida, pero con una mediana diversidad de formas de vida según cobertura. Su fisonomía es de bosque multiestratificado. M ontaldo (1974) también diferencia como unidad fitosociológica a sitios dominados por $N$. dombeyi, A. araucana y $N$. pumilio.

La unidad Np-A a posee 44 especies en la RNM, lo que representa el $20,9 \%$ de su flora, con un bajo porcentaje de especies introducidas. A demás, es la que posee una similitud de especies promedio mayor, lo que se explicaría por su posición media en el gradiente altitudinal, lo cual permite que se presenten especies tanto de las partes superiores como inferiores de este gradiente. Presenta el segundo valor más alto de diversidad de especies por forma de vida, pero con una mediana diversidad de formas de vida según cobertura. Su fisonomía es de bosque multiestratificado. Este tipo de comunidad también ha sido descrita por Oberdorfer (1960), Montaldo (1974) y Gajardo (1994).

La unidad Np-Bb posee 38 especies (18\%), de las cuales el $5,3 \%$ son introducidas. Presenta una mediana diversidad de especies por forma de vida, y su diversidad de formas de vida según cobertura es baja. Esto último se debe a la alta dominancia de la macrofanerófita lenga, y una baja cobertura de arbustos y hierbas. Corresponde a una fisonomía de bosque achaparrado de $\mathrm{K}$ rumholz de lenga. La diferenciación de esta comunidad como unidad fitosociológica particular también ha sido propuesta por San Martín \& Ramírez (1987) y San Martín et al. (1991). No obstante, con el fin de verificar si corresponden a la misma unidad fitosociológica, habría que evaluar la variación latitudinal de la composición florística de este tipo de comunidad debido a la gran amplitud latitudinal que posee.

$\mathrm{L}$ a unidad $\mathrm{Na}$ - $\mathrm{Pp}$ posee una riqueza de 21 especies $(10 \%)$, siendo la menos rica de todas las unidades, pero, sin embargo, sin la presencia de especies introducidas. También presenta uno de los menores valores de similitud promedio, por lo cual esta unidad conformaría un singular grupo florístico. Posee un valor medio de diversidad de especies por forma de vida, pero es una de las unidades de menor diversidad de formas de vida según cobertura, debido a una alta dominancia de la macrofanerófita Nothofagus antarctica (ñirre), y una baja abundancia de nanofanerófitos y en general de las otras formas de vida. Su fisonomía es de bosque achaparrado correspondiente a K rumholz. Este tipo de unidad, donde domina fuer- temente el ñirre, ya ha sido diferenciada por Oberdorfer (1960), San Martín \& Ramírez (1987) y San Martín et al. (1991), entre otros. Sin embargo, la amplia distribución geográfica que posee podría determinar diferencias florísticas considerables, por lo cual es necesario analizar si todas éstas corresponden a la misma unidad fitosociológica.

La unidad A a-Fs posee una riqueza de 34 especies $(16,1 \%)$, sin especies introducidas. Presenta una mediana diversidad de especies por forma de vida, mayor que otras unidades multiestratificadas como $\mathrm{Na}-\mathrm{Lp}$, pero sin embargo una mediana diversidad de formas de vida según cobertura. Presenta una fisonomía de bosque biestratificado, con un estrato arbóreo dominado sólo por Araucaria araucana, y un sotobosque bajo principalmente herbáceo. Esta unidad ya ha sido diferenciada por Oberdorfer (1960) y Gajardo (1994) como una unidad fitosociológica florísticamente particular.

$L$ a unidad DC-Be es la unidad que posee la más alta riqueza de especies (58) con el $27,5 \%$ de la flora de la RNM y además un bajo porcentaje de especies introducidas. Presenta una baja diversidad de especies por forma de vida, dada por la alta riqueza relativa de caméfitas y hemicriptófitas respecto de las demás, pero un valor medio de diversidad de formas de vida según cobertura. Posee una fisonomía de estepa arbustiva altoandina, dominada por caméfitas arbustivas con el $70 \%$ de la cobertura vegetacional.

$\mathrm{L}$ a unidad $\mathrm{C}$ a-A o posee una riqueza de 43 especies $(20,4 \%)$, con un bajo porcentaje de introducidas. Presenta una mediana diversidad de especies por forma de vida, debido a la ausencia de formas de vida macrofanerófita, y es la unidad de menor diversidad de formas de vida según cobertura, debido a la alta dominancia de nanofanerófítas con el $87,5 \%$ de la cobertura vegetacional, especialmente por Chusquea argentina. Por esto presenta una fisonomía de matorral. Esta unidad podría ser de tipo secundaria, resultante de incendios, naturales 0 antropogénicos, o de algún otro tipo de perturbaciones de la vegetación original, no obstante no presenta una alta invasión de especies introducidas. En este caso, Chusquea argentina sería un arbusto colonizador que cobra gran importancia con una alta densidad cuando se elimina el estrato arbóreo, impidiendo la regeneración de $N$. antarctica, $N$. pumilio 0 A. araucana que son las especies arbóreas que se distribuyen en los mismos ambientes donde se presenta esta 
unidad. Esto también se sustenta en los valores medios a altos de similitud de especies que posee esta unidad con las que distribuyen en este tipo de ambiente. En consecuencia, esta unidad podría provenir tanto de las unidades $\mathrm{Np}-\mathrm{Bb}, \mathrm{Na}-\mathrm{Pp} O$ A a-Fs. Este patrón también ha sido documentado por Ramírez et al. (1991), en donde otras especies de Chusquea dominan sectores donde la vegetación original ha sido eliminada.

La unidad A e-Ps posee una riqueza de 22 especies $(10,4 \%)$, siendo una de las menos ricas de la RNM, aunque con un bajo porcentaje de introducidas. Además presenta una muy baja similitud florística promedio con las demás unidades, conformando un grupo singular de especies. Debido a la alta riqueza relativa de hemicriptófitas respecto a las demás formas de vida, posee una baja diversidad de especies por forma de vida, siendo una de las más bajas de la RNM. También posee un bajo valor de diversidad de formas de vida según cobertura debido a la alta dominancia de caméfitas subarbustivas, tales como Adesmia emarginata. Posee una fisonomía de estepa herbácea altoandina.

La unidad $\mathrm{Gm}$-Em posee una riqueza de 22 especies $(10,4 \%)$, siendo una de las de menor riqueza entre las unidades, pero también con una baja invasión de especies introducidas. Es la unidad con menor similitud promedio, lo cual se debería a su carácter de comunidad azonal de vegas al toandinas, las cuales poseen en general una composición florística particular y diferenciada de la vegetación zonal (Villagrán et al. 1983). Posee una alta riqueza relativa de geófitos, lo que hace que sea la unidad de menor diversidad de especies por forma de vida, y una alta abundancia de hemicriptófitas y geófitas con $56,1 \%$ y $42,6 \%$ de la cobertura vegetacional respectivamente, lo que determina su bajo valor de diversidad de formas de vida según cobertura. Posee una fisonomía de pradera herbácea. Las comunidades dominadas por Gunnera magellanica ya han sido diferenciadas por Pisano (1977) y Villagrán (1980) en distintas zonas del sur de Chile. Por esto, se requiere analizar su variabilidad florística para establecer si corresponden a la misma unidad fitosociológica.

Las diferencias de diversidad alfa entre las unidades, expresadas en diferentes porcentajes de riqueza de especies, debieran ser consecuencia principalmente de distintas tasas de especiación a nivel regional entre los diferentes ambientes en que se presentan las unidades. También es posible que a ciertas unidades se haya dispersado, posterior a los períodos glaciales, un mayor número de especies respecto a otras. La inmigración de especies introducidas contribuye considerablemente a la riqueza de algunas unidades, mientras que en otras no. A la escala espacial de cada unidad vegetacional, este tipo de factores regionales e históricos serían más importantes que factores más locales, tales como exclusión por competencia, en determinar la variación de riqueza de especies entre las unidades vegetacionales (Ricklefs \& Schluter 1993).

Las diferencias composicionales 0 de diversidad beta entre las unidades está relacionada con la altitud. Las unidades de los extremos altitudinales, ya sea bajo 0 alto, son las que poseen grupos más particulares de especies, con una menor similitud promedio con el resto de las unidades. Además, las unidades de los pisos medios presentan una mayor similitud promedio, lo que se debería a que poseen especies compartidas tanto con los pisos superiores como inferiores.

Por otro lado, las unidades presentan diferente diversidad de formas de vida, tanto en riqueza de especies como cobertura. Esto sería atribuible a las diferentes posiciones topográficas y con ello de las condiciones de temperatura y humedad, las cuales son determinantes tanto del número de especies como de la cobertura de las formas de vida que se pueden desarrollar en un sitio determinado (Raunkiaer 1937; Danin \& Orshan 1990).

En conclusión, las diferencias estructurales de especies y formas de vida entre las unidades sugiere que la definición de unidades vegetacionales fitosociológicas es una estrategia eficiente para representar toda la flora de un área en diferentes unidades de vegetación, y que la incorporación de un mayor número de éstas dentro del SNASPE permitiría representar en él una mayor diversidad florística y biológica en general. Esto debido a que las unidades de vegetación, al poseer una diferente composición florística, debieran presentar, en mayor o menor grado, distintos tipos de organismos y procesos ecosistémicos (Noss 1987).

\section{AGRADECIMIENTOS}

Se agradece a CONAF de Curacautín (IX Región) por el financiamiento y facilidades logísticas durante el período de muestreo. Además, se agradece a M arco Sepúlveda, estudiante de Ingeniería Forestal de la Universidad de Chile, por colaborar en el muestreo de la vegetación. 


\section{BIBLIOGRAFIA}

ARMESTO ，」., J. GUTIERREZ， J. MARTINEZ. 1979. "Las comunidades vegetales de la región mediterránea de Chile: distribución de especies y formas de vida en un gradiente de aridez", Medio Ambiente 4 (1): 62-70.

BARKMAN, J., J. MORAVEC, S. RA USCHERT. 1986. "Code of phytosociological nomenclature", Vegetatio 67: 145-195.

BECERRA, P., L. FAUNDEZ. 1999. Diversidad Florística de Ia Reserva Nacional Malalcahuello, IX Región de Chile. Chloris chilensis 2(1), http://www.chlonschile.cl

BENOIT, I. 1996. Representatividad ecológica del sistema nacional de areas protegidas del Estado. En: MUÑOZ, M., H. NUÑEZ, J. YAÑEZ (eds), Libro Rojo de los Sitios Prioritarios para la Conservación de la Diversidad Biológica. Corporación Nacional Forestal, Santiago, Chile.

BRAUN-BLANQUET, J. 1950. Sociología Vegetal; estudio de las comunidades vegetales. Buenos Aires: A cme A gency, $421 \mathrm{p}$

DANIN, A., G. ORSHAN. 1990. "The distribution of Raunkiaer life forms in Israel in relation to the environment", Journal of Vegetation Science 1: 41-48.

DI CASTRI F., E. HAJEK. 1976. Bioclimatología de Chile. Ediciones Universidad Católica de Chile, $128 \mathrm{p}$.

FORMAN, R. 1995. Land Mosaics. The ecology of landscapes and regions. Cambridge University Press, $632 \mathrm{p}$.

GAJARDO, R. 1980. Vegetación del bosque de Araucaria araucana (Mol.) K. Koch. en la Cordillera de los Andes (Lonquimay). Bol. Técnico $\mathrm{N}^{\circ}$ 58, Fac. Cs. Forestales, Universidad de Chile.

GAJARDO, R. 1994. La Vegetación Natural de Chile. Clasificación y Distribución Geográfica. Edit. Universitaria, Chile. $165 \mathrm{p}$.

HILDEBRAND, R., R. GODOY, A. VOGEL. 1990. "Subantarctic-A ndean Nothofagus pumilio forests", Vegetatio 89: 55-68.

HOWE, H., J. SMALLWOOD. 1982. "Ecology of seed dispersal", Annual Review of Ecology and Systematic 13: 201-228.

KREBS, C. 1989. Ecological Methodology. New Y ork: Harper $\&$ Row Publishers, $654 \mathrm{p}$

LA NDRUM , L., T. NIM LOS. 1975. Gradientes florales y morfología asociada del suelo en la Reserva Forestal de Malalcahuello. Boletín técnico $\mathrm{N}^{\circ} 35, \mathrm{Fac}$. Cs. Forestales Universidad de Chile.

LARA, A., C. DONOSO, J. ARAVENA. 1995. La conservación del bosque nativo en Chile problemas y desafíos. En: ARMESTO, J., VILLAGRAN, C., ARROYO, MK. (eds.) Ecología de los Bosques Nativos de Chile. Edit. Universitaria, $470 \mathrm{p}$

LUEBERT， F., P. BECERRA . 1998. "Representatividad vegetacional del sistema nacional de áreas silvestres protegidas del Estado (SNASPE) en Chile", Ambiente y Desarrollo 14(2): 62-69.

MARTICORENA, C., M. QUEZADA. 1985. "Catalogo de la flora vascular de Chile", Gayana Botánica 42(1-2): 1-158.

M ONTALDO, P. 1974. "La Bioecologia de Araucaria araucana (M ol.) K och". Bol. Ins, Forestal Latino Americano de Invest. y Capac. Venezuela 46/48: 3-55.

M ULLER-DOMBOIS, D., H. ELLENBERG. 1974. Aims and methods of vegetation ecology. N ew Y ork: Edit. J. Wiley y Sons, $460 \mathrm{p}$.

NOSS, R. 1987. "From Plant Communities to Landscapes in Conservation Inventories: A look at the $\mathrm{N}$ ature Conservancy (USA)", Biological Conservation 41: 11-37.

NOSS, R. 1990. "Indicators for Monitoring Biodiversity: A Hierarchical Approach", Conservation Biology 4: 355-364.
OBERDORFER, E. 1960. "Pflanzensoziologische Studien in Chile. Ein Vergleich mit Europa", Flora et Vegetatio Mundi 2: 1-208.

PERALTA, M. 1980. Geomorfología, clima y suelos del tipo forestal A raucana en Lonquimay. Boletín técnico $N^{\circ} 57$. Fac. Cs. Forestales, Universidad de Chile.

PISA N O, E. 1977. "Fitogeografía de la Fuego-Patagonia chilena, I. Comunidades vegetales entre las latitudes $52^{\circ}$ y $56^{\circ} "$, Anales del Instituto de la Patagonia 8: 121-250.

POLLMANN, W. 2000. Sintaxonomía y Ecología del Dasyphyllo. Nothofagion alpinae en Chile. XII Reunión A nual de la Sociedad Botánica de Chile, XXVII Jornadas Argentinas de Botánica. Gayana Botánica 57.

RAMIREZ, C. 1978. "Estudio florístico y vegetacional del Parque Nacional Tolhuaca (Malleco-Chile)", Publicación ocasional del Museo de Historia Natural, Santiago, 24: 3-23.

RAMIREZ, C., R. WESTERMEIER. 1976. "Estudio de la vegetación espontánea del Jardín Botánico de la Universidad Austral de Chile (Valdivia), como ejemplo de tabulación fitosociológica", AgroSur 4: 93-105.

RAMIREZ.C., F. FERRIERE, H. FIGUEROA. 1983. "Estudio fitosociológico de los bosques pantanosos templados del sur de Chile", Revista Chilena de Historia Natural 56: 5772.

RAMIREZ, C., H. FIGUEROA. 1987. "Fitosociología de los Nothofagus de la zona higromórfica chilena", Bosque 8(2): 127-132.

RAMIREZ, C., E. HAUENSTEIN, D. CONTRERAS, J. SAN MARTIN. 1988. "Degradación antrópica de la vegetación en la depresión intermedia de la A raucanía, Chile", AgroSur 16: 1-14.

RAMIREZ, C., C. SAN MARTIN, H. FIGUEROA, R. MAC DONALD, V. FERRADA. 1991. "Estudios ecosociológicos en la vegetación de los ñadis de la décima región de Chile", AgroSur 19: 34-47.

RAUNKIAER, C. 1937. Plant Life Forms. London: Oxford University Press, $104 \mathrm{p}$.

RICKLEFS, R., R. SCHLUTER. 1993. Species Diversity: Regional and Historical Influences. En: Ricklefs, R.; R Schluter (eds.) Species Diversity in Ecological Communities. Historical and Geographical Perspectives. University of Chicago Press, $416 p$

SAN MARTIN, J., H. FIGUEROA, C. RAMIREZ. 1984. "Estudio fitosociológico de los bosques de Ruil (Nothofagus alessandrii Espinosa) en Chile Central", Revista Chilena de Historia Natural 57: 171-200.

SAN MARTIN, J., A. TRONCOSO, C. RAMIREZ. 1986. "Fitosociología de los bosques de Nothofagus antarctica (Forst.) Oesrt. en la cordillera costera de Cauquenes ( $\mathrm{Chi}$ le)", Bosque 7(2): 65-78.

SAN MARTIN, J., C. RAMIREZ. 1987. "Fitosociología de los Nothofagus de la zona mesomórfica chilena", Bosque 8(2): 121-125.

SAN MARTIN, J., A. TRONCOSO, A. MESA, T. BRAVO, C. RAMIREZ. 1991. "Estudio Fitosociológico del bosque caducifolio magallánico en el limite norte de su área de distribución", Bosque 12(2): 29-41.

SANTIBAÑEZ, F. 1993. A tlas A groclimático de Chile, IX región. Ministerio de Agricultura, Fondo de Investigación A graria, CORFO. Stgo.

SCHMITHÜSEN, J. 1956. "Die Räumliche Ordnung Chilenischen Vegetation", Bonn. Geogr. Abh (17): 1-86.

SOLLINS, P. 1998. "Factors influencing species composition in tropical lowland rain forest does soil matter?", Ecology 79: 23-30.

STEPHENSON, N. 1990. "Climatic control of Vegetation distribution: the role of water balance", American Naturalist 135: 649-670. 
TILMAN, D. 1988. Plant Strategies and the Dynamics and Structure of Plant Communities. Princeton University Press, USA.

VEBLEN, T., T. KITZBERGER, B. BURNS, A. REBERTUS. 1995. Perturbaciones y dinámica de regeneración en bosques andinos del sur de Chile y A rgentina. En: ARMESTO, J.; VILLAGRAN, C; ARROYO, MK. (eds.), Ecología de los Bosques Nativos de Chile. Edit. Universitaria, 470 p.

VEIT, H., K. GARLEFF. 1995. Evolución del paisaje cuaternario y los suelos en Chile central-sur. En: ARMESTO, J.; VILLAGRAN, C; ARROYO, MK. (eds.), Ecología de los Bosques Nativos de Chile. Edit. Universitaria, $470 \mathrm{p}$.

VILLAGRAN, C. 1980. "Vegetationsgeschichtliche und Pflanzensoziologische Untersuchungen im Vicente Pérez Rosales Nationalpark, Chile", Dissertationes Botanicae 54: 1-165.
VILLAGRAN, C, M. T. K. ARROYO, C. MARTICORENA. 1983. "Efectos de la desertización en la distribución de la flora andina de Chile", Revista Chilena de Historia Natural 56: $137-157$

VILLAGRAN, C, F. HIN OJOSA. 1997. "Historia de los bosques del sur de Sudamérica, II: A nálisis fitogeográfico", Revista Chilena de Historia Natural 70: 241-267.

VILLASEÑOR, R. 1980. "Unidades fisionómicas y florísticas del Parque Nacional La Campana", Anales del Museo de Historia Natural de Valparaíso 13: 65-70.

WESSER, S., S. ARM BRUSTER. 1991. "Species distribution controls across a forest-stepe transition: a causal model and experimental test", Ecological Monographs 6: 323-342.

WHITTAKER, R. 1972. "Evolution and measurement of species diversity", Taxon 21: 213-251.

WHITTAKER, R. 1973. Handbook of Vegetation Science. Netherlands: W. Junk, The Hague. 


\section{APÉNDICE 1}

Composición y cobertura relativa de las especies de cada unidad vegetacional de la R NM.

Composition and relative coverage of species in the vegetation units of RNM.

\begin{tabular}{|c|c|c|c|c|c|c|c|c|c|c|c|c|c|c|}
\hline \multirow{2}{*}{ Especies } & \multirow{2}{*}{$\begin{array}{c}\text { Forma } \\
\text { de } \\
\text { vida }\end{array}$} & \multicolumn{13}{|c|}{ Unidades Vegetacionales Fitosociológicas } \\
\hline & & $\mathrm{Ao}-\mathrm{Au}$ & Na-Lp & $\mathrm{Nd}-\mathrm{Fm}$ & $\mathrm{Na}-\mathrm{Nd}$ & $\mathrm{Np}-\mathrm{Nd}$ & $\mathrm{Np}-\mathrm{Bb}$ & $\mathrm{Np}-\mathrm{Aa}$ & $\mathrm{Na}-\mathrm{Pp}$ & $\mathrm{Dc}-\mathrm{Be}$ & $\mathrm{Aa}-\mathrm{Fs}$ & $\mathrm{Ca}-\mathrm{Ao}$ & Ae-Ps & $\mathrm{Gm}-\mathrm{Em}$ \\
\hline Acuena leptacantha & $\mathrm{C}$ & & & & & & 0,264 & & & 1,270 & & & 3,240 & \\
\hline Acaena ovalifolia & $\mathrm{C}$ & 13,368 & 0,280 & 0,540 & 0,230 & 0,380 & & 0,950 & & & & 0,570 & & 0,040 \\
\hline Acaena pinnatifida & $\mathrm{C}$ & 0,307 & & & & & 0,132 & 0,005 & 0,144 & 0,763 & & 0,539 & 3,239 & \\
\hline Achillea millefolium* & $\mathrm{H}$ & 5,839 & 0,094 & & 0,059 & 0,005 & & & & & & & & \\
\hline Adenocaulon chilense & $\mathrm{H}$ & & 0,188 & & 0,117 & 0,479 & 0,792 & 2,400 & & & 0,344 & & & \\
\hline Adesmia emarginata & C & & & & & & & & & 0,254 & 0,344 & & 69,231 & \\
\hline Agrostis capillaris* & $\mathrm{H}$ & & 0.375 & 0,145 & 0,012 & & & & & & & & & \\
\hline Agrostis philippiana & G & & & & & & & & 0,029 & & & & & 0,041 \\
\hline Agrostis uliginosa & $\mathrm{H}$ & 6,453 & & & & & & & & & & & & 0,415 \\
\hline Nstroemeria aurantiaca & G & & 0,188 & 0,657 & 0,176 & 0,190 & & 0,185 & & & & & & \\
\hline $\begin{array}{l}\text { Alstroemeria patagonica } \\
\text { Amphibromus scabriuscula }\end{array}$ & $\begin{array}{l}\mathrm{G} \\
\mathrm{G}\end{array}$ & & & & & & 0,396 & & & & & 0,539 & & \\
\hline & $\mathrm{G}$ & & & & & 0,000 & & & & 0,509 & 0.017 & & & \\
\hline Anthoxanthum juncifolia* & $\mathrm{H}$ & & & & & & & & & & & 0,198 & & \\
\hline Anthoxanthum odoratum & $\mathrm{H}$ & 1,260 & & & & & & & & & & & & \\
\hline Apium sp. & $\mathrm{H}$ & & & & $=$ & & & 0,136 & & & & & & \\
\hline Araucaria araucana & $\mathrm{M}$ & & & & & 5,049 & & 19,321 & 0,433 & & 62,285 & & & \\
\hline Arentaria serpens & $\mathrm{H}$ & & & & & & & & & & & & 1,619 & \\
\hline Armeria maritima & $\mathrm{C}$ & & & & & & & & & 0,051 & & & & \\
\hline Aster vahli & $\mathrm{H}$ & & & & & & & & & & & & & 0,415 \\
\hline Asteranthera ovata & $\mathrm{L}$ & & 0,488 & & & & & & & & & & & \\
\hline Azara alpina & N & & & & & 0,237 & & 1,830 & & & .. & & & \\
\hline Azara lanceolata & $\mathrm{N}$ & & 0,938 & 2,498 & 0,357 & 0,427 & & & & & & & & \\
\hline Azara mycrophylla & $\mathrm{N}$ & 0,307 & 0,094 & & 0,176 & & & & & & & & & \\
\hline Azorella monantha & $\mathrm{C}$ & & & & & & & & & 0,509 & & & & \\
\hline Baccharis magellanica & $\mathrm{C}$ & & & & & & 2,376 & & 0,144 & 1,527 & 0344 & 0,180 & 0,810 & \\
\hline Baccharis obovata & $\mathrm{N}$ & & & & & & & & 0.866 & 1,527 & 0,344 & 1258 & & \\
\hline Berberis buxifolia & $N$ & & & & & 0,142 & 1,056 & 0,407 & & & & 1,238 & & 0,415 \\
\hline Berberis darwinii & $\mathrm{N}$ & & 0,188 & 0,131 & 0,351 & & & & & 11,198 & 0.172 & 0.539 & & \\
\hline $\begin{array}{l}\text { Berberis empetrifolia } \\
\text { Berberis linearifolia }\end{array}$ & $\begin{array}{l}\mathrm{C} \\
\mathrm{N}\end{array}$ & & 0,197 & & 0,182 & 0,100 & 0,396 & 0,090 & & & & & & \\
\hline Berberis serrato-dentuta & $\mathrm{N}$ & 0,307 & 0,094 & $0,13 \mathrm{I}$ & 0,293 & 0,332 & & 0,090 & & & & & & \\
\hline Blechnum blechnoides & G & & 0,751 & 0,526 & 0,117 & 0,005 & & & & & & & & \\
\hline Blechnum chilense & G & & & 0,263 & & & & & & & & & & \\
\hline Blechnum microphyllum & G & & & & & 0,095 & & 0,045 & & & & & & \\
\hline Blechnum pennamarina & G & & & & 0,064 & 0,100 & & 0,045 & & & & 0,180 & & \\
\hline Bromus burkartii & G & & & & & & 0,132 & & & & & & & \\
\hline Bromus lithobius & $\mathrm{H}$ & 5,839 & & 0,013 & & & & & & & & & & \\
\hline Calandrinia affinis & G & & & & & & & & & & & & 0,810 & \\
\hline Calandrinia dianthoides & $\mathrm{H}$ & & & & & & & & & 0,254 & & & & \\
\hline Calandrinia hirtella & $\mathrm{H}$ & & & & & & & , & & & & & 0,891 & \\
\hline Calceolaria biflora & $\mathrm{H}$ & & & & & 0,005 & & & & 0,280 & & & 0,810 & \\
\hline Calceolaria cavanillesi & $\mathrm{H}$ & & & & & & & & & & 0,172 & 0,018 & & \\
\hline Calceolaria foliosa & $\mathrm{H}$ & & & 0,039 & & & & & & & & & & \\
\hline Calceolaria sp. & $\mathrm{H}$ & & & & & & & & 0.014 & & & 0,018 & & \\
\hline Caltha sagitata & $\mathrm{G}$ & & & & & & & 0,090 & 0,014 & & & & & 2,075 \\
\hline Cardamine cordata & $\mathrm{H}$ & & 0,188 & 0,131 & 0,059 & 0,152 & & & & & & & & \\
\hline $\begin{array}{l}\text { Cardamine tenuirostris } \\
\text { Carex aphylla }\end{array}$ & $\begin{array}{l}\mathrm{H} \\
\mathrm{H}\end{array}$ & & & & & & 2,112 & & 0,144 & & & $\begin{array}{l}0,018 \\
0,180\end{array}$ & & \\
\hline Carex decidua & G & & & & & & & & & & & & & 1,245 \\
\hline Carex lateriflora & G & & & & & & & 0,050 & 4,761 & & & & & \\
\hline Centaurea cyanus* & $\mathrm{T}$ & & & 0,394 & & & & & & & & & & \\
\hline Cerastium arvense* & $\mathrm{H}$ & & & 0,013 & & & & & & 0,305 & & 0,054 & 0,081 & \\
\hline Chaetanthera lanata & G & & & & & & & & & & & & 1,619 & \\
\hline Chiliotrichum rosmarinifolit & $\operatorname{ium} \mathrm{N}$ & & & & & & & 0,090 & 0,433 & 0,509 & & & & \\
\hline Chloraea gaudichaudii & $\mathrm{H}$ & & & & & & & & & & 0,017 & & & \\
\hline
\end{tabular}


Apéndice (continuación)

\begin{tabular}{|c|c|c|c|c|c|c|c|c|c|c|c|c|c|c|}
\hline \multirow{2}{*}{ Especies } & \multirow{2}{*}{$\begin{array}{c}\text { Forma } \\
\text { de } \\
\text { vida }\end{array}$} & \multicolumn{13}{|c|}{ Lnidades Vegelacionales Fitosociológicas } \\
\hline & & Ao-Au & $\mathrm{Na}-\mathrm{Lp}$ & $\mathrm{Nd}-\mathrm{Fm}$ & $\mathrm{Na}-\mathrm{Nd}$ & $\mathrm{Np}-\mathrm{Nd}$ & $\mathrm{Np}-\mathrm{Bb}$ & $\mathrm{Np}-\mathrm{Aa}$ & $\mathrm{Na}-\mathrm{Pp}$ & $\mathrm{Dc}-\mathrm{Be}$ & Aa-Fs & Ca-Ao & Ae-Ps & $\mathrm{Gm}-\mathrm{Em}$ \\
\hline Chloraea magellanica & $\mathrm{H}$ & & & & & & & & & 0,025 & & & & \\
\hline Chloraea sp. & $\mathrm{H}$ & & & & & & 0,026 & 0,005 & & & & & & \\
\hline Chrysosplenium valdivianum & $m G$ & & & 2,367 & & & & & & & & & & \\
\hline Chusquea argentina & $\mathrm{N}$ & & 16,999 & 4,076 & 32,349 & 23,160 & & 23,660 & 27,918 & & 3,608 & 85,336 & & \\
\hline Coriaderia pilosa & $\mathrm{H}$ & & & & & & & & & & & & & 0,415 \\
\hline Crepis capillaris* & $\mathrm{T}$ & & & 0,053 & & & & & & & & 0,036 & & \\
\hline Cynanchum nummularifoliun & & & & & & & 0,132 & & & 0,534 & 0,017 & & & \\
\hline Cynosurus echinatus & $\mathrm{T}$ & 5,839 & & & & & & & & & & & & \\
\hline Cystopteris fragilis & $\mathrm{G}$ & & & 0,263 & & 0,005 & & 0,005 & & & & & & \\
\hline Dactylis glomerata* & $\mathrm{H}$ & 1,537 & & & 0,059 & & & & & & & & & \\
\hline Dasyphyllum diacanthoides & $M$ & & 4,925 & & & & & & & & & & & \\
\hline Deschampsia sp. & G & & & & & & & & & & & & & 1,245 \\
\hline Dioscorea andina & $\mathrm{L}$ & & & & & $\begin{array}{l}0,009 \\
0,024\end{array}$ & & & & & & & & \\
\hline Dioscorea brachybotrya & $\mathrm{L}$ & & 0,009 & & 0,129 & 0,024 & & & & & & & & \\
\hline Discaria chacaye & $\mathrm{C}$ & & & & & & & & & 33,975 & 2,062 & 1,078 & & \\
\hline Drimys andina & $\mathrm{N}$ & & & & 2,547 & 10,999 & & & & & & & & \\
\hline Dysopsis glechomoides & $\mathrm{H}$ & & 0,094 & & 0,006 & 0,095 & & & & & & & & 0,415 \\
\hline Eleocharis albibracteata & $\mathrm{G}$ & & & & & & & & & & & & & 1,245 \\
\hline Eleocharis melanostachys & G & & & & & & & & & & & & & 33,402 \\
\hline Elymus andinus & $\mathrm{H}$ & & & & & & & & & & & 0,180 & & \\
\hline Elymus antarctica & G & & & & & & & & & 0,025 & & & & \\
\hline Embothrium coccineum & $\mathrm{N}$ & & & & 1,054 & 0,142 & 0,396 & & & 0,763 & & & & \\
\hline Empetrum rubrum & $\mathrm{C}$ & & & & & & 0,132 & & & 8,398 & 1,031 & & & \\
\hline Ephedra andina & $\mathrm{N}$ & & & & & & & & 0,014 & 0,254 & 0,172 & & & \\
\hline $\begin{array}{l}\text { Epilobium ciliatum } \\
\text { Epilobium densifolium }\end{array}$ & $\begin{array}{l}\mathrm{G} \\
\mathrm{G}\end{array}$ & & & & & & & & & 0,254 & & & 0,081 & \\
\hline Equisetum bogotense & G & & & 0,013 & & & & & & & & & & \\
\hline Erigeron cinereus & $\mathrm{H}$ & & & & & & & & & 0,051 & & & & \\
\hline Erigeron leptopetalus & G & & & & & & & & & 0,280 & & & & \\
\hline Escallonia virgata & $\mathrm{N}$ & & & & & & 0,792 & & 0,433 & 0,763 & 0,344 & 0,539 & & \\
\hline Escallonia alpina & $\mathrm{N}$ & & & & & & & & & 1,527 & & & & \\
\hline Euphorbia collina & $\mathrm{H}$ & & & & & & & & & 0,102 & & 0,018 & 0,972 & \\
\hline Euphrasia chrysantha & $\mathrm{H}$ & & & & & 0,047 & & & & 0,025 & 0,017 & & & \\
\hline Festuca scabriuscula & $\mathrm{H}$ & & & & & & 0,541 & 1,717 & 0,592 & 4,326 & 22,852 & 0,557 & & \\
\hline Fragaria chiloensis & C & & & & 0,117 & 0,142 & 2,376 & 0,181 & 0,289 & & 0,172 & 1,078 & & \\
\hline Fuchsia magellanica & $N$ & & 0,094 & 43,394 & & & & & & & & & & \\
\hline Galium fuegianum & $\mathrm{H}$ & & & & & & & & 0,043 & & 0,172 & & & \\
\hline Gaultheria phillyreifolia & $\mathrm{N}$ & & 0,094 & 0,263 & 0,293 & 0.522 & & & & & & & & \\
\hline Geranium magellanicum & $\mathrm{H}$ & & & 0,394 & & 0,052 & & & & & & & & 0,415 \\
\hline Geum magellanicum & $\mathrm{G}$ & & & & & & & & & & & & & 0,083 \\
\hline Gunnera tinctoria & $\mathrm{N}$ & & & 0,789 & & & & & & & & & & \\
\hline Gunnera magellanica & $\mathrm{H}$ & & & & & & & & & & & & & $\begin{array}{r}53,942 \\
0415\end{array}$ \\
\hline Hamadryas magellanica & $\mathrm{G}$ & & & & & & & 0,181 & & 0,254 & & 0018 & & 0,415 \\
\hline $\begin{array}{l}\text { Haplopappus grindelioides } \\
\text { Hediptis }\end{array}$ & $\begin{array}{l}\mathrm{C} \\
\mathrm{H}\end{array}$ & 1260 & & & & & & & & & & & & \\
\hline $\begin{array}{l}\text { Hediotis salzmannit } \\
\text { Hieracium pilosela* }\end{array}$ & $\begin{array}{l}\mathrm{H} \\
\mathrm{H}\end{array}$ & 1,200 & & & & & 0,132 & & & & & & & \\
\hline Hypochoeris radicata* & $\mathrm{H}$ & 0,338 & 0,103 & 0,026 & 0,012 & & & & & & & & & \\
\hline Hypochoeris tenuifolia & $\mathrm{H}$ & & & & & & & & & 0,305 & & 0,036 & & \\
\hline Juncus acutus & $\mathrm{H}$ & & & 0,408 & & & & & & & & & & \\
\hline lagenophora hirsuta & G & & & & & 0,095 & 0,132 & & & & & & & \\
\hline Lapsana comunis* & $\mathrm{T}$ & 6,453 & & 0,013 & 0,006 & & & & & & & & & \\
\hline Lathyrus magellanica & $\mathrm{L}$ & & & & & & 0,132 & 0,045 & & 0,534 & & & & \\
\hline Lathyrus multiceps & L & & & & & 0,005 & & & & & 0,344 & 0,719 & & \\
\hline Laureliopsis philippiana & M & & 23,595 & & & & & & & & & & & \\
\hline Leontodon taraxacoides* & $\mathrm{H}$ & 6,453 & & & & & & & & & & & & \\
\hline Leucheria magna & $\mathrm{H}$ & & & & & 0,005 & & & & & & & & \\
\hline Libertia sp. & G & & & & & & & & & & & & & 0,830 \\
\hline Licopodium magellanicum & G & & & & & & 0,013 & $0,27 \mathrm{I}$ & & & & & & \\
\hline Loasa argentina & $\mathrm{H}$ & & 0,009 & & & & & & & & & & & \\
\hline Lomatia hirsufa & $\mathrm{M}$ & & & & 0,176 & & & & & & & & & \\
\hline Luzula chilensis & $\mathrm{H}$ & & & & & & & & & 0,025 & 0,017 & & & \\
\hline Macrachaenium gracile & $\mathrm{G}$ & & & & & 0,114 & & 0,045 & & & & & & \\
\hline
\end{tabular}


Apéndice (continuación)

\begin{tabular}{|c|c|c|c|c|c|c|c|c|c|c|c|c|c|c|}
\hline \multirow{2}{*}{ Especies } & \multirow{2}{*}{$\begin{array}{c}\text { Forma } \\
\text { de } \\
\text { vida }\end{array}$} & \multicolumn{13}{|c|}{ Unidades Vegetacionales Fitosociológicas } \\
\hline & & $\mathrm{Ao}-\mathrm{Au}$ & $\mathrm{Na}-\mathrm{Lp}$ & $\mathrm{Nd}-\mathrm{Fm}$ & $\mathrm{Na}-\mathrm{Nd}$ & $\mathrm{Np}-\mathrm{Nd}$ & $\mathrm{Np}-\mathrm{Bb}$ & $\mathrm{Np}-\mathrm{Aa}$ & $\mathrm{N}_{\mathrm{a}-\mathrm{Pp}}$ & $\mathrm{Dc}-\mathrm{Be}$ & Aa-Fs & $\mathrm{Ca}-\mathrm{AO}$ & Ae-Ps & $\mathrm{Gm}-\mathrm{Em}$ \\
\hline Maytenus disticha & $\mathrm{N}$ & & & & 1,171 & 9,695 & 1,716 & 9,017 & & & 0,687 & & & \\
\hline Maytenus magellanica & $\mathrm{N}$ & & & & 0,234 & & & 0,181 & & & & & & \\
\hline Mimulus luteus & $\mathrm{T}$ & & & 0,920 & & & & & & & & & & \\
\hline Misodendrum punctulatum & $P$ & & & & & 0,095 & & 0,271 & & & & & & \\
\hline Mulinum spinosum & $\mathrm{N}$ & & & & & & & & & 1,781 & 0,344 & 0,359 & 4,858 & \\
\hline Mutisia decurrens & $\mathrm{L}$ & & & 0,013 & & & & & & & & & & \\
\hline Myoschitos oblonga & $\mathrm{N}$ & & 0,094 & 0,131 & & 0,147 & 0,145 & 0,235 & & & $0,36 \mathrm{r}$ & ; & & \\
\hline Myrceugenia ovata & $\mathrm{N}$ & & 0,281 & 0,263 & 0,059 & & & & & & & & & \\
\hline Myrceugenia planipes & N & & & & 0,176 & & & & & & & & & \\
\hline Nassauvia aculeata & $\mathrm{C}$ & & & & & & & & & 0,509 & & 0,198 & & \\
\hline Nassauvia lagascae & $\mathrm{H}$ & & & & & & & & & & & & 0,891 & \\
\hline Nertera granadensis & $\mathrm{H}$ & & 0,094 & & & 0,005 & & & & & & & & \\
\hline Nothofagus alpina & M & & 28,379 & 9,468 & 36,330 & & & & & & & & . & \\
\hline Nothofagus antarcrica & $\mathrm{M}$ & & & & & & & & 59,515 & & & & & \\
\hline Nothofagus dombeyi & $\mathrm{M}$ & & 16,418 & 27,614 & 20,726 & 22,544 & & & & & & &. & \\
\hline Nothofagus obliqua & $\mathrm{M}$ & 12,446 & & & & & & & & & & & & \\
\hline Nothofagus pumilio & $\mathrm{M}$ & & & & & 22,093 & 80,839 & 28,134 & & & & & & \\
\hline Oreopolus glacialis & $\mathrm{C}$ & & & & & & & & & 0,051 & & & & \\
\hline Osmorhyza chilensis & $\mathrm{H}$ & & 0,375 & 0,526 & 0,468 & 0,384 & 0,396 & 0,452 & & & 0,172 & & & \\
\hline Oxalis adenophila & G & & & & & & & & & & & & 1,619 & \\
\hline Oxalis valdiviensis & $\mathrm{T}$ & & & 0,145 & 0,006 & & & & & & & & & \\
\hline Perezia pedicularifolia & G & & & & & & 0,264 & 0,050 & 0,014 & 0,305 & 0,515 & & & \\
\hline Perezia pilifera & $\mathrm{G}$ & & & & & & & & & 0,509 & & & & \\
\hline Perezia prenanthoides & $\mathrm{G}$ & & & & & 0,047 & & 0,045 & & & & & & \\
\hline Pernettya myrtilloides & $\mathrm{N}$ & & & & & & 0,924 & 8,610 & & 0,509 & & & & \\
\hline Pernettya pumila & $\mathrm{C}$ & & & & & & & & 2,597 & 5,853 & 1,375 & 0,359 & & \\
\hline Phacelia secunda & $\mathrm{H}$ & & & & & & 0,264 & & & 0,280 & & 0,359 & 3,320 & \\
\hline Phleum pratense* & $\mathrm{H}$ & & & & 0,006 & & & & & & & & & \\
\hline Plantago australis & $\mathrm{C}$ & 2,766 & & & & & & & & & & & & \\
\hline Plantugo lanceolata* & $\mathrm{H}$ & & & & 0,059 & . & & & & & & & & \\
\hline Poa alopecurus & $\mathrm{H}$ & & & & & & & & & 8,678 & 0,017 & 0,198 & & \\
\hline Poa andina & $\mathrm{G}$ & & & & & & & & 0,721 & & & & & \\
\hline Poa pratensis* & $\mathrm{G}$ & 5,839 & & & & & & & & & & & . & \\
\hline Poa sp. & $\mathrm{H}$ & & & & & & & & & & 0,052 & 0,180 & & \\
\hline Poa trivialis* & $\mathrm{H}$ & 5,531 & & & & & & . & & & & & & \\
\hline Polystichum plicatum & $\mathrm{G}$ & & & 0,026 & 0,006 & 0,052 & & " & & & & & & \\
\hline Polystichum subintegerrimun & $n \mathrm{G}$ & & & & 0,006 & 0,047 & & & & & & & & \\
\hline Pozoa volcanica & $\mathrm{H}$ & & & & & & & & & 0,331 & & 0,359 & 0,810 & \\
\hline Prunella vulgaris* & $\mathrm{H}$ & 1,537 & & 0,131 & 0,064 & & & & & & & & & \\
\hline Pseudopanax laetevirens & $\mathrm{M}$ & & 0,094 & 0,394 & 0,059 & & & & & & & & & \\
\hline Quinchamalium chilense & $\mathrm{H}$ & & & & & & & & & 0,560 & $0,24 !$ & & & \\
\hline Ranunculus chilensis & $\mathrm{H}$ & 0,031 & & & & & & & & & & & & \\
\hline Ranunculus peduncularis & $\mathrm{H}$ & & & & & & 0,013 & & & 0,611 & & & & \\
\hline Rhodophiala andina & $\mathrm{G}$ & & & & & & & & & 1,043 & & & 1,700 & \\
\hline Ribes cucullatum & $\mathrm{C}$ & & & & & & 0,792 & 0,095 & & & & 1.258 & & 0,830 \\
\hline Ribes magellanicum & $\mathrm{C}$ & & & & & & & & 0,866 & & 0,344 & & & \\
\hline Ribes punctatum & $\mathrm{N}$ & & & & 0,117 & & & & & & & & & \\
\hline Ribes trilobum & $\mathrm{N}$ & & 3,893 & 0,394 & 0,176 & & & & & & & & & \\
\hline Ribes valdivianum & $\mathrm{N}$ & & 0,188 & 0,657 & 0,703 & & & & & & & & & \\
\hline Rosa moschata* & $\mathrm{N}$ & 5,531 & & & & & & & & & & & & \\
\hline Rubus geoides & $\mathrm{C}$ & & & & & & 0,396 & 0,009 & & & & & & \\
\hline Rumex acetosella* & $\mathrm{H}$ & 1,537 & 0,281 & 0,131 & 0,117 & 0,047 & 0,132 & 0,050 & & 0,534 & & 0,180 & 2,429 & \\
\hline Rytidosperma picta & $\mathrm{H}$ & & & & & & & & & 0,254 & & & & \\
\hline Senecio argyreus & $\mathrm{C}$ & & & & & & & & & 0,509 & & & 0.810 & \\
\hline Senecio baccharidifolites & $\mathrm{C}$ & & & & & & & & & 0,763 & & & & \\
\hline Senecio fistulosus & $\mathrm{G}$ & & & & & & & & & & & & 0.415 & \\
\hline Senecio hieracium & $\mathrm{G}$ & & & & & & & & & & & 0,036 & 0,415 & \\
\hline Senecio linearifolius & $\mathrm{C}$ & & & & & & & & & 0,280 & & 0,198 & & \\
\hline Senecio otites & $\mathrm{G}$ & & & & 0,006 & & & & & & & & & \\
\hline Senecio phylicifolius & $\mathrm{C}$ & & & & & & & & & & & 0,018 & & \\
\hline Senecio pilquensis & $\mathrm{C}$ & & & & & & 0,264 & & & & & & & \\
\hline Senecio poepigii & $\mathrm{C}$ & & & & & & 0,396 & & & & & & & \\
\hline
\end{tabular}


Apéndice (continuación)

\begin{tabular}{|c|c|c|c|c|c|c|c|c|c|c|c|c|c|c|}
\hline \multirow{2}{*}{ Especies } & \multirow{2}{*}{$\begin{array}{c}\text { Forma } \\
\text { de } \\
\text { vida }\end{array}$} & \multicolumn{13}{|c|}{ Unıdades Vegetacionales Fitosociológicas } \\
\hline & & Ao-Au & $\mathrm{Na}-\mathrm{Lp}$ & $\mathrm{Nd}-\mathrm{Fm}$ & $\mathrm{Na}-\mathrm{Nd}$ & $\mathrm{Np}-\mathrm{Nd}$ & $\mathrm{Np}-\mathrm{Bb}$ & $\mathrm{Np}-\mathrm{Aa}$ & $\mathrm{Na}-\mathrm{Pp}$ & $\mathrm{Dc} \cdot \mathrm{Be}$ & $\mathrm{Aa}-\mathrm{Fs}$ & $\mathrm{Ca}-\mathrm{Al}_{0}$ & Ac-Ps & Gm-Em \\
\hline Senecio subpubescens & C & & & & & & 0,026 & & & & & & & \\
\hline Senecto subumbellatus & $\mathrm{C}$ & & & & & & 0,158 & & 3,334 & & 0,180 & 0,081 & & \\
\hline Seneclo tristis & $\mathrm{C}$ & & & & & & & & & 0,025 & & & & \\
\hline Silene andicola & $\mathrm{H}$ & & & & & 0,047 & & & & & & & & \\
\hline Sisyrinchium arenarium & $G$ & & & & & & & & & 0,305 & & & & \\
\hline Sisyrinchium chilense & $\mathrm{G}$ & & & & & 0,005 & 0,290 & 0,136 & & 0,025 & & 0,557 & 0,081 & \\
\hline Sisyrinchum patagonicum & $\mathrm{G}$ & & & & & & & & & & 0,172 & & & \\
\hline Solanum cyrtopodium & $\mathrm{N}$ & & 0,094 & & 0,123 & & & & & & & & & \\
\hline Solanum etuberosum & $\mathrm{G}$ & & & 0,657 & & & & & & & & & & \\
\hline Solenomelus stsyrinchum & $G$ & & & & & & & & & & & 0,539 & & \\
\hline Solidago chilensts & G & & & & & & & & & & & 0,018 & & \\
\hline Stellana sp. & $\mathrm{H}$ & & & 0,013 & & 0,047 & & 0,045 & & & & & & \\
\hline Stipa laevissima & $\mathrm{H}$ & 5,531 & & & & & & & & & & & & \\
\hline Stipa sp. & $G$ & & & & & & & & & & & 1,078 & & \\
\hline Taraxacum officulane* & $\mathrm{H}$ & 1,537 & 0,009 & 0,013 & 0,293 & & & & & & & & & \\
\hline Trifollum repens* & $\mathrm{C}$ & 2,151 & 0,094 & 0,131 & & & & & & & & & & \\
\hline Trisetum sclerophyllum & $\mathrm{H}$ & & & & & & . & & & 0,254 & & & & \\
\hline Tropaeolum speciosum & $\mathrm{H}$ & & 0,009 & 0,013 & 0,006 & & & & & & & & & \\
\hline Uncinia brevicaults & $\mathrm{G}$ & & & & & & & 0,136 & & & & & & 1,245 \\
\hline Unctmia erinaceae & $\mathrm{G}$ & & & & & & 0,132 & 0,045 & & & & & & \\
\hline Uncinia lechlenana & $G$ & & & & & & & 0,090 & & & 0,172 & & & \\
\hline Uncina phleotdes & $\mathrm{G}$ & & & & & 0,052 & & & & & & & & \\
\hline Valeriana carnosa & $\mathrm{H}$ & & & & & & & & & 0,789 & & & & \\
\hline Valeriana hebecarpa & $\mathrm{H}$ & & & & & 0,047 & & 0,050 & 0,029 & & & & & \\
\hline Verbascum thapsus* & $\mathrm{H}$ & & & 0,013 & 0,006 & & & & & & + & & & \\
\hline Verbena sp. & $\mathrm{H}$ & & & & & & & & & 0,254 & & & & \\
\hline Veronica serphyllifolia* & $\mathrm{H}$ & & & 0,131 & & & & & & & & & & 0,041 \\
\hline Vicia nigricans & $\mathrm{L}$ & & 0,009 & 0,145 & 0,076 & 0,341 & & 0,185 & & & & & & \\
\hline Vola dasyphilla & $\mathrm{G}$ & & & & & & 0,396 & & & 0,254 & & 0,018 & & \\
\hline Viola magellanica & . $\mathrm{H}$ & & & 0,526 & 0,064 & 1,185 & & 0,321 & & & 0,017 & & & \\
\hline Viola reichei & $\mathrm{G}$ & & & & & & & & & & & 0,018 & & \\
\hline
\end{tabular}

* Especie introducida.

Las especies no determinadas taxonómicamente (sp) no fueron consideradas en los análisis de especies introducidas. 